\title{
CLASSIFYING TORSION-FREE SUBGROUPS OF THE PICARD GROUP
}

BY

ANDREW M. BRUNNER, MICHAEL L. FRAME,

YOUN W. LEE AND NORBERT J. WIELENBERG

\begin{abstract}
Torsion-free subgroups of finite index in the Picard group are the fundamental groups of hyperbolic 3-manifolds. The Picard group is a polygonal product of finite groups. Recent work by Karrass, Pietrowski and Solitar on the subgroups of a polygonal product make it feasible to calculate all the torsion-free subgroups of any finite index. This computation is carried out here for index 12 and 24 , where there are, respectively, 2 and 17 nonisomorphic subgroups. The manifolds are identified by using surgery.
\end{abstract}

1. Introduction. The Picard group $\operatorname{PSL}(2, Z(i))$ is the projective special linear group of $2 \times 2$ matrices over the complex integers. Alternatively, it is the (discrete) group of linear fractional transformations $\{z \rightarrow(a z+b) /(c z+d)\}$ where $a, b, c, d$ are complex integers and $a d-b c=1$. A linear fractional transformation of $\mathbf{C}$ extends to an isometry of hyperbolic 3-space $\mathbf{H}^{3}=\{z+t j: z \in \mathbf{C}, j=(0,0,1)\}$. Consequently, a torsion-free subgroup of the Picard group acts freely on $\mathbf{H}^{3}$ and the quotient space is a hyperbolic 3-manifold. If the subgroup is also of finite index, the manifold has finite hyperbolic volume and can be described as "the complement of a link in a closed 3-manifold". In this paper, we study the torsion-free subgroups for the two smallest possible indices, i.e., index 12 or 24 , and the topology of the associated manifolds.

The method of producing torsion-free subgroups is an application of results of A. Karrass, A. Pietrowski, and D. Solitar [2] on the subgroups of a polygonal product of groups. These results describe the structure of a polygonal product in terms of conjugates of edge and vertex groups, where the conjugating elements are doublecoset representatives in a connected, compatible, regular, extended Schreier system (hereafter called a " $c$-cress").

In our calculations we obtain (up to isomorphism) two subgroups of index 12 and seventeen subgroups of index 24; this classifies the torsion-free subgroups of index 24 , and (up to homeomorphism) the hyperbolic 3-manifolds which can be obtained in this way. We describe the geometry and topology of those associated manifolds. This algebraic method of producing 3-manifolds with hyperbolic structure gives

Received by the editors January 12, 1983. The paper was presented at the Special Session on Four-Dimensional Topology, 804th Meeting at the A.M.S., Salt Like City, April 29-30, 1983, University of Utah.

1980 Mathematics Subject Classification. Primary 57M25, 20F05, 30F40; Secondary 20F34, 57S30. 
many interesting examples which do not appear to be directly accessible using geometric-topological methods. In principle, the method produces all torsion-free subgroups of finite index.

The listing and reduction of cases required extensive hand and machine computation, and is not reproduced here. A permutation representation is given for each subgroup. From this follows a presentation for the group and a faithful representation in the Picard group.

The authors wish to thank A. Karrass, A. Pietrowski, and D. Solitar for having made available to them unpublished material, and for their very generous assistance; three of the authors have visited York University for consultation at their invitation. Financial assistance from the National Research Council of Canada and the University of Wisconsin-Parkside is gratefully acknowledged.

2. The Picard group as a polygonal product. The following is the Karrass-SolitarPietrowski polygonal product presentation of the Picard group (hereafter called Pic). There are generators

and relations

$$
\begin{array}{rlrl}
a_{2} & =\left(\begin{array}{cc}
0 & i \\
i & 1
\end{array}\right), & b_{14} & =\left(\begin{array}{cc}
0 & 1 \\
-1 & 0
\end{array}\right), \\
c_{21}=\left(\begin{array}{cc}
0 & i \\
i & 0
\end{array}\right), & d_{4}=\left(\begin{array}{cc}
1 & 1 \\
-1 & 0
\end{array}\right),
\end{array}
$$

$$
\begin{gathered}
a_{2}^{3}=b_{14}^{2}=c_{21}^{2}=d_{4}^{3}=1, \\
\left(a_{2} d_{4}\right)^{2}=1, \quad a_{2} b_{14}=b_{14} a_{2}^{2}, \\
d_{4} c_{21}=c_{21} d_{4}^{2}, \quad b_{14} \sim c_{21},
\end{gathered}
$$

where here $x \sim y$ means $x$ commutes with $y$. This presentation can be verified from well-known presentations (e.g., Swan [7]). We are especially interested in the following four subgroups (these will be vertex groups):

$$
\begin{aligned}
& A=\left\langle a_{2}, a_{4}=d_{4} ; a_{2}^{3}=a_{4}^{3}=\left(a_{2} a_{4}\right)^{2}=1\right\rangle, \\
& B=\left\langle b_{14}, b_{2}=a_{2} ; b_{2}^{3}=b_{14}^{2}=\left(b_{2} b_{14}\right)^{2}=1\right\rangle, \\
& C=\left\langle c_{21}, c_{14}=b_{14} ; c_{14}^{2}=c_{21}^{2}=\left(c_{14} c_{21}\right)^{2}=1\right\rangle, \\
& D=\left\langle d_{4}, d_{21}=c_{21} ; d_{4}^{3}=d_{21}^{2}=\left(d_{4} d_{21}\right)^{2}=1\right\rangle .
\end{aligned}
$$

Note that $A \cong A_{4}, B \cong D \cong S_{3}$ and $C \cong V_{4}$. Pic can be presented as a polygonal product by placing these four groups at the vertices of a square and certain cyclic subgroups on the edges, as shown

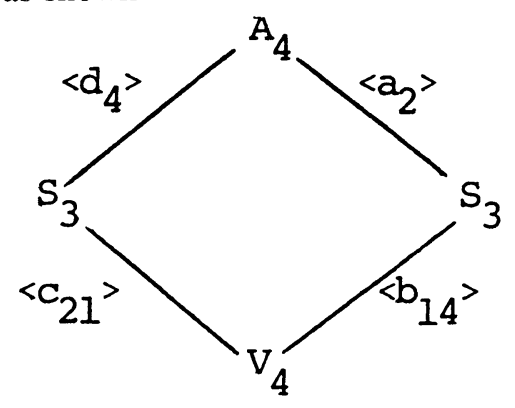


Any two consecutive vertices and the joining edge form a free product with amalgamation (f.p.a.). Pic is itself a f.p.a. of the f.p.a. along one edge with the f.p.a. along the opposite edge, amalgamating the free product of the cyclic groups lying on the remaining two edges. (Here $b_{14}$ and $d_{4}$ generate the modular group $\operatorname{PSL}(2, Z)$ ).

The subgroup theorem of Karrass, Pietrowski and Solitar states that a subgroup $H$ of Pic is obtained as a certain factor group of the $H N N$ group with base a tree product of groups having as vertices the intersection of $H$ with conjugates of vertex groups of Pic and edges the intersection of $H$ with conjugates of edges of Pic; the additional relations are due to the polygon "closing up", and involve only the stable letters in the $H N N$ group (the $t$-symbols). Now a torsion-free subgroup cannot intersect a conjugate of a vertex or edge group; in particular, the minimal index for such a group is 12 . Thus, in the presentation for a torsion-free subgroup the base group collapses, leaving only the $t$-symbols and relations between them.

3. From $c$-cress to subgroup. In order to find all torsion-free subgroups of index $n$ we find all regular representations of Pic onto transitive subgroups of $S_{n}$. Here, regular means that if $v$ is in a conjugate of a vertex group, then its representative in $S_{n}$ cannot have a fixed point (if we consider this representation to be defined by right multiplication on the right cosets of $H$, and if $(H x) v=H x$, then $x v x^{-1} \in H$; but $H$ does not intersect conjugates of vertices nontrivially). Hence what is required are all possible sets of choices of $a_{2}, b_{14}, c_{21}$ and $d_{4}$ in $S_{n}$ which (i) satisfy the relations of Pic, (ii) generate a transitive subgroup of $S_{n}$, and (iii) ensure that the representative of an element in a vertex group does not fix a point. Each torsion-free subgroup $H$ corresponds to such a representation with $H$ equal to the preimage of those elements which fix 1.

From a representation of Pic in $S_{n}$, a presentation for $H$ is produced from a $c$-cress. An outline of this technique follows. (The reader is referred to Karrass and Solitar [1, §5, p. 239] for more details of notation.)

There are vertex groups $A, B, C, D$. Elements of these groups are called vertex symbols, or $\alpha, \beta, \gamma, \delta$ symbols, respectively. Elements of edge groups are called edge symbols. The square is given an orientation

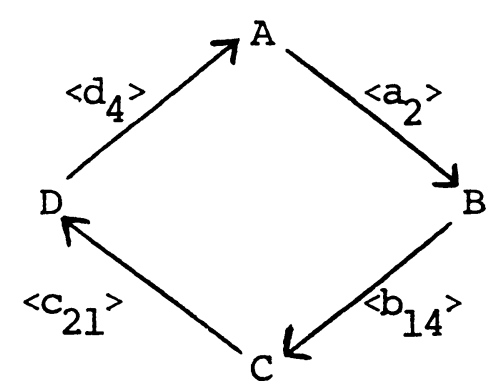

A Schreier system is a set of right coset representatives for which any initial segment of a representative is again a representative.

Extended means there is a collection of (in this case, four) kinds of right coset representatives (called $A$ reps, $B$ reps, $C$ reps, $D$ reps) such that whatever kind of symbol a representative ends in, it is that kind of representative; and if the last symbol is deleted, the resulting element is another representative of the same kind. 
Regular means that if the final $\alpha$ symbols are deleted from all the $A$ reps, the resulting words form an $(H, A)$ double coset representative system, and similarly for the other vertices.

Compatible means that when the edge symbols are deleted completely from the ends of the $A$ reps, the resulting words form an $(H, U)$ double coset representative system, where $U$ is the edge group which follows $A$, and similarly for the other edges.

Connected means that if an $X$ rep ends in a $y$ symbol, the $X$ rep must also be in the representative systems of every vertex on the oriented path from $Y$ to $X$.

A presentation for $H$ is obtained as follows. Suppose ${ }^{\alpha} K,{ }^{\beta} K,{ }^{\gamma} K,{ }^{\delta} K$ are the $A$, $B, C, D$, representatives, respectively, of the same given coset. The generators for $H$ are

$$
\begin{array}{ll}
t_{\alpha_{\kappa}}={ }^{\alpha} K^{\beta} K^{-1}, & t_{\beta_{K}}={ }^{\beta} K^{\gamma} K^{-1}, \\
t_{\gamma_{K}}={ }^{\gamma} K^{\delta} K^{-1}, & t_{\delta_{K}}={ }^{\delta} K^{\alpha} K^{-1},
\end{array}
$$

where $K$ ranges over all cosets of $H$. There are three kinds of relations.

Type 1. $t_{\alpha_{K}} t_{\beta_{K}} t_{\gamma_{K}} t_{\delta_{K}}=1$.

Type 2. $t_{\varepsilon_{K}}=t_{\varepsilon_{\|K\|}}$ where ${ }^{\varepsilon}\|K\|$ is the ${ }^{\varepsilon} K$ with edge symbols deleted.

Type 3. $t_{\varepsilon_{K}}=1$ if ${ }^{\varepsilon} K$ and ${ }^{\varepsilon+1} K$ are freely equal, where $\varepsilon$ and $\varepsilon+1$ refer to consecutive vertices in the given orientation.

4. A clarifying example. The notation is chosen to be as mnemonic as possible. Let $H$ be a torsion-free subgroup of index 12 in Pic, and let $a_{1}=$ identity of Pic, $a_{3}=a_{2}^{2}, a_{5}=a_{4}^{2}, a_{6}=a_{4} a_{2}^{2}, a_{7}=a_{2} a_{4}^{2}, a_{8}=a_{4}^{2} a_{2}, a_{9}=a_{2}^{2} a_{4}, a_{10}=a_{2} a_{4}, a_{11}=$ $a_{4} a_{2}, a_{12}=a_{2} a_{4}^{2} a_{2}$ be the elements of the vertex group $A$. Then the 12 distinct cosets of $H$ are $H a_{1}, H a_{2}, H a_{3}, \ldots, H a_{12}$. Therefore, a regular transitive representation of Pic in $S_{12}$ associated with $H$ can be obtained using the 1-1 correspondence $H a_{i} \rightarrow i, i=1,2,3, \ldots, 12$. Up to conjugation in $S_{12}$, we may therefore choose

$$
\begin{aligned}
& a_{2}=\left(\begin{array}{lll}
1 & 2 & 3
\end{array}\right)(4116)(5810)(1297), \\
& a_{4}=d_{4}=\left(\begin{array}{lll}
14 & 4
\end{array}\right)(1286)(2107)(3911) .
\end{aligned}
$$

In order to find all torsion-free subgroups of index 12 in Pic (up to isomorphism), it remains to make choices for $b_{14}$ and $c_{21}$, keeping $a_{2}$ and $d_{4}$ fixed as above.

A possible choice for $b_{14}$ and $c_{21}$ is

$$
\begin{aligned}
& b_{14}=\left(\begin{array}{l}
4 \\
1
\end{array}\right)\left(\begin{array}{l}
6 \\
2
\end{array}\right)(87)(109)\left(\begin{array}{ll}
11 & 3
\end{array}\right)(125), \\
& c_{21}=\left(\begin{array}{ll}
3 & 1
\end{array}\right)\left(\begin{array}{l}
7 \\
6
\end{array}\right)(82)(95)(114)(1210) .
\end{aligned}
$$

We describe how to obtain the presentation for the subgroup associated with it.

A $c$-cress for this representation is given in Table 1 .

REMARK. The numbers at the left refer to cosets. A coset is determined by where a representative sends the number 1 . The edge representatives are used first to fill in slots of the form $11 x$. Then vertex representatives are used to fill in remaining slots, working in the direction of orientation of the square and maintaining the required properties of the $c$-cress. This is an algorithmic process, but is difficult to describe in complete detail. The subgroup theorem guarantees the existence of a $c$-cress. 
TABLE 1

\begin{tabular}{|l|lll|lll|llll|lll|}
\hline & & $A$ & & \multicolumn{3}{|c|}{$B$} & & & $C$ & \multicolumn{3}{|c|}{$D$} \\
\hline 1. & 1 & 1 & 1 & 1 & 1 & 1 & 1 & 1 & 1 & 1 & 1 & 1 \\
2. & 1 & 1 & $a_{2}$ & 1 & $b_{2}$ & 1 & $b_{2}$ & 1 & 1 & $b_{2}$ & 1 & 1 \\
3. & 1 & 1 & $a_{3}$ & 1 & $b_{3}$ & 1 & 1 & 1 & $c_{21}$ & 1 & $d_{21}$ & 1 \\
4. & 1 & $a_{4}$ & 1 & 1 & 1 & $b_{14}$ & 1 & $c_{14}$ & 1 & 1 & 1 & $d_{4}$ \\
5. & 1 & $a_{5}$ & 1 & $a_{5}$ & 1 & 1 & $a_{5}$ & 1 & 1 & 1 & 1 & $d_{5}$ \\
6. & 1 & $a_{4}$ & $a_{3}$ & 1 & $b_{2}$ & $b_{14}$ & $b_{2}$ & $c_{14}$ & 1 & $b_{2}$ & $d_{21}$ & $d_{4}$ \\
7. & 1 & $a_{7}$ & 1 & $a_{5}$ & $b_{2}$ & $b_{14}$ & $b_{2}$ & $c_{14}$ & $c_{21}$ & $b_{2}$ & 1 & $d_{5}$ \\
8. & 1 & $a_{5}$ & $a_{2}$ & $a_{5}$ & $b_{2}$ & 1 & $b_{2}$ & 1 & $c_{21}$ & $b_{2}$ & $d_{21}$ & 1 \\
9. & 1 & $a_{7}$ & $a_{3}$ & $a_{5}$ & $b_{3}$ & $b_{14}$ & $a_{5}$ & 1 & $c_{21}$ & 1 & $d_{21}$ & $d_{4}$ \\
10. & 1 & $a_{5}$ & $a_{3}$ & $a_{5}$ & $b_{3}$ & 1 & $a_{5}$ & $c_{14}$ & $c_{21}$ & $b_{2}$ & 1 & $d_{4}$ \\
11. & 1 & $a_{4}$ & $a_{2}$ & 1 & $b_{3}$ & $b_{14}$ & 1 & $c_{14}$ & $c_{21}$ & 1 & $d_{21}$ & $d_{5}$ \\
12. & 1 & $a_{7}$ & $a_{2}$ & $a_{5}$ & 1 & $b_{14}$ & $a_{5}$ & $c_{14}$ & 1 & $b_{2}$ & $d_{21}$ & $d_{5}$ \\
\hline
\end{tabular}

Using the three kinds of relations, many of the generators become trivial. Many nontrivial generators become equal in pairs or triples by relations of type 2 . This is indicated by lining out; the remaining generators are shown in Table 2. The remaining relations can be reduced by working first with relators of length 2 , and then of length 3 .

$$
\begin{array}{llll}
t_{3} t_{9}=1 & \Rightarrow & t_{9}=\bar{t}_{3} \\
t_{1} t_{6}=1 & \Rightarrow & t_{6}=\bar{t}_{1} \\
t_{1} t_{7} t_{10}=1 & \Rightarrow & t_{7}=\bar{t}_{1} \bar{t}_{10} \\
t_{2} t_{4} t_{7}=1 & \Rightarrow & \Rightarrow & \\
t_{4} t_{10}=1 & t_{2}=\bar{t}_{7} \bar{t}_{4}=\bar{t}_{1} t_{4} \\
t_{2} t_{5} t_{9}=1 & \Rightarrow & \Rightarrow & t_{2}=\bar{t}_{4} \bar{t}_{1} \bar{t}_{4} \\
t_{5} t_{8}=1 & \Rightarrow & t_{10}=\bar{t}_{4} \\
t_{1} t_{3} t_{6} t_{9}=1 & \Rightarrow & \\
t_{2} t_{8} t_{10}=1 & &
\end{array}
$$

TABLE 2

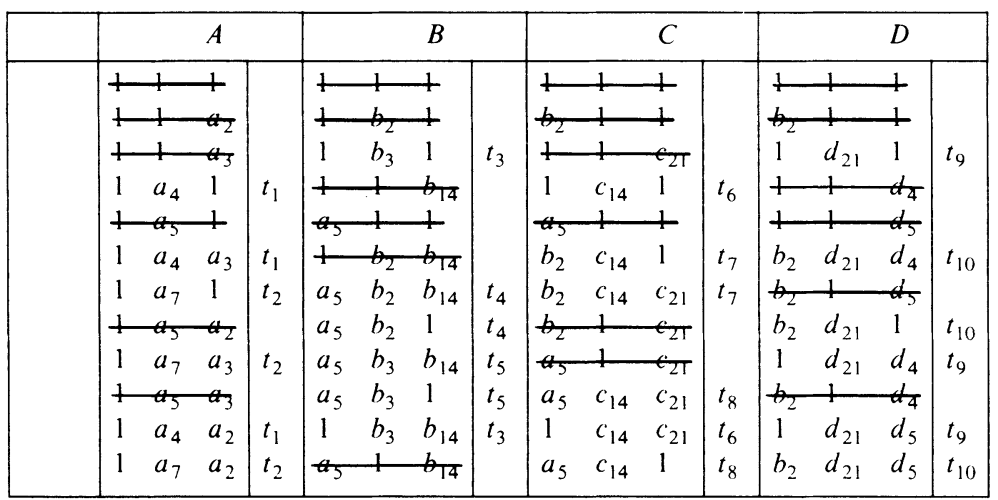


The presentation simplifies to $\left\langle t_{1}, t_{3}, t_{4} ; t_{1} \sim t_{3}, t_{3}=\bar{t}_{4} t_{1} \bar{t}_{4}^{3} t_{1} \bar{t}_{4}\right\rangle$.

An annoying confusion arises from the fact that the $c$-cress produces words which are read left to right in the link groups, whereas the words in Pic with matrix multiplication read from right to left. This does not matter in the above presentation, which is symmetric, but we note carefully that with matrix multiplication $t_{1}=b_{14}^{-1} a_{4}$, for example, rather than $a^{-1} b_{14}^{-1}$. A matrix representation for this group is

$$
\begin{aligned}
& t_{1}=b_{14}^{-1} a_{4}=\left(\begin{array}{ll}
1 & 0 \\
1 & 1
\end{array}\right), \\
& t_{3}=d_{21}^{-1} b_{3}=\left(\begin{array}{ll}
1 & 0 \\
i & 1
\end{array}\right), \\
& t_{4}=b_{2}^{-1} d_{21}^{-1} b_{2} a_{5}=\left(\begin{array}{cc}
2 i & 1+2 i \\
1 & 1-i
\end{array}\right),
\end{aligned}
$$

5. Topology of the quotient manifold. A complete hyperbolic 3-manifold $M$ with finite volume is homeomorphic to $N-l$ where $N$ is a closed 3-manifold and $l$ is a disjoint union of finitely many copies of $S^{1}$. Equivalently, $M$ is the interior of a compact 3-manifold $\bar{M}$ with finitely many boundary components, each of which is a torus [8]. In addition, $\bar{M}$ is irreducible and the boundary components are incompressible. The maximal abelian subgroups of $\pi_{1}(\bar{M})$ are isomorphic to either $\mathbf{Z}$ or $\mathbf{Z} \oplus \mathbf{Z}$. Each $\mathbf{Z} \oplus \mathbf{Z}$ subgroup is peripheral, i.e., up to choice of basepoint it is induced by the inclusion mapping of a boundary torus into $\bar{M}$. So there is a conjugacy class of peripheral subgroups for each boundary component of $\bar{M}$.

The matrix representatives of a peripheral subgroup are parabolics (trace $= \pm 2$ ). Conversely, the parabolic generators of a peripheral subgroup can be conjugated in $\operatorname{PSL}(2, \mathbf{C})$ to be of the form $\left(\begin{array}{ll}1 & 1 \\ 0 & 1\end{array}\right)$ and $\left(\begin{array}{ll}1 & \tau \\ 0 & 1\end{array}\right)$, where $\operatorname{Im} \tau>0$. This gives a Euclidean structure on the associated boundary torus (see Thurston [8]).

Mostow's Rigidity Theorem says that if two complete hyperbolic finite-volume 3-manifolds have isomorphic fundamental groups, then there is an isometry which induces the isomorphism. Consequently, the set of $\tau$ 's of the boundary components of $\bar{M}$ provide topological invariants of $M$. Each $\tau$-invariant is determined up to a choice of generators for the peripheral subgroup. So $\tau_{1}$ and $\tau_{2}$ are equivalent if

$$
\tau_{2}=\frac{m \tau_{1}+n}{p \tau_{1}+q} \quad \text { for some }\left(\begin{array}{cc}
m & n \\
p & q
\end{array}\right) \in \operatorname{SL}(2, \mathbf{Z}) .
$$

A fundamental region for the action of $\operatorname{PSL}(2, \mathbf{Z})$ on the upper half-plane $\{\tau$ : $\operatorname{Im} \tau>0\}$ is given by $|\tau| \geqslant 1,-\frac{1}{2} \leqslant \operatorname{Re} \tau \leqslant \frac{1}{2}$. In addition, if the isometry is allowed to be orientation-reversing, then $\tau_{1}$ and $\tau_{2}$ correspond to equivalent Euclidean structures if $\tau_{1}=-\bar{\tau}_{2}$.

The manifolds corresponding to the subgroups are described in $\$ 10$ by surgery instructions on links in $S^{3}$. A theorem of Waldhausen [9] implies that a compact, irreducible, sufficiently large 3-manifold whose fundamental group is isomorphic to one of these subgroups is homeomorphic to the closure of the hyperbolic 3-manifold. In $\$ 11$ we check that these conditions hold.

A fundamental polyhedron for the action of a group $H$ on $\mathbf{H}^{3}$ is useful for understanding the group and the manifold. We are most interested in Ford domains. 
This is obtained by conjugating $H$ if necessary so that one of the parabolic fixed points is $\infty$. The stabilizer of $\infty$ is then generated by two Euclidean translations $z \rightarrow z+w_{1}$ and $z \rightarrow z+w_{2}$ and a $\tau$-invariant of this boundary component is $w_{1} / w_{2}$ or $w_{2} / w_{1}$. If $\left(\begin{array}{ll}a & b \\ c & d\end{array}\right) \in H$ with $c \neq 0$, the isometric hemispheres of this transformation are $|(z+t j)--d / c|=1 /|c|$ and $|(z+t j)-a / c|=1 /|c|$. A Ford domain is a fundamental polyhedron for $H$ which consists of those points which lie above all the isometric hemispheres of $H$ and inside some fundamental region for the stabilizer of $\infty$. By sufficiently patient computation of isometric hemispheres, this description can be used to produce a Ford domain. A Ford domain has totally geodesic faces which are subsets either of planes perpendicular to $\mathbf{C}$, or of hemispheres centered in C. (See papers of Riley [5] and Wielenberg [10] for more details and examples.)

A Ford domain for a subgroup of Pic is typically a union of hyperbolically convex polyhedra, with some or all of the vertices "at infinity" on the boundary of $\mathbf{H}^{3}$. These ideal vertices are parabolic fixed points with each cycle of ideal vertices corresponding to a boundary torus of the manifold. The peripheral subgroups of $H$ can be found from the cycles of ideal vertices. A presentation for $H$ can be derived from the cycles of edges.

6. The example, continued. The transformations $t_{1}$ and $t_{3}$ generate a peripheral subgroup fixing 0 . The transformations $t_{3}^{-1} t_{1} t_{4}^{-1}$ and $t_{1} t_{4} t_{1}^{-1} t_{4} t_{3}$ are parabolics fixing $i$. Conjugation by $\left(\begin{array}{cc}i & 0 \\ 1 & -i\end{array}\right)$ moves these fixed points to 0 and $\infty$. Then

$$
\begin{gathered}
t_{1}=\left(\begin{array}{cc}
1 & 0 \\
-1 & 1
\end{array}\right), \quad t_{3}=\left(\begin{array}{cc}
1 & 0 \\
-i & 1
\end{array}\right), \\
t_{3}^{-1} t_{1} t_{4}^{-1}=\left(\begin{array}{cc}
1 & -1-2 i \\
0 & 1
\end{array}\right), \quad t_{1} t_{4} t_{1}^{-1} t_{4} t_{3}=\left(\begin{array}{cc}
1 & -2+i \\
0 & 1
\end{array}\right) .
\end{gathered}
$$

A Ford domain with sides paired by these transformations, (actually its projection into the complex plane) is given in Figure 1. There are two nonequivalent ideal vertices so the quotient manifold $M$ has two boundary tori. Also $t_{1}^{-1} t_{3}$ and $t_{4} t_{1}^{-1} t_{3}$ lie in different peripheral subgroups and are each primitive elements of that subgroup. Filling in solid tori to kill these elements trivializes the group. So $M$ is the complement of a two-component link in a homotopy 3 -sphere.

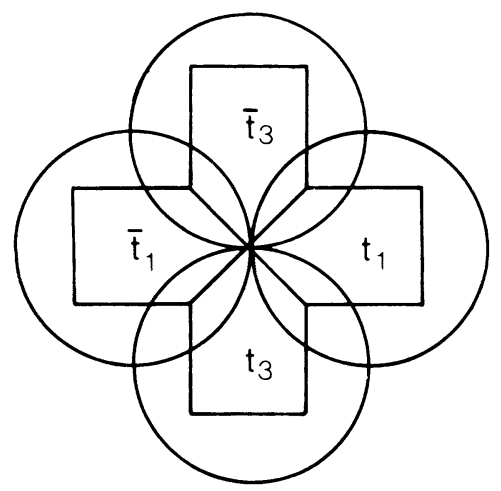

Figure 1 
Furthermore, filling in one component at a time produces quotient groups $\mathbf{Z}$ (reverse the order of the letters and let $t_{3} t_{1}^{-1} t_{4}=1$ ) and the fundamental group of the trefoil (let $t_{1}^{-1} t_{3}=1$ ). We look for a link in $S^{3}$ with one component an unknot and the other a trefoil. The $\tau$-invariant for each component is $i$.

When dealing with words arising from the Ford domain above, it should be remembered with the order of the letters is reversed from what they were before. Inspection shows that $t_{3} t_{1}^{-1}$ and $t_{1}$ generate one peripheral subgroup, while $t_{3} t_{1}^{-1} t_{4}$ and $t_{3} t_{4} t_{1}^{-1} t_{4} t_{1}\left(=t_{1}^{-1} t_{4}^{2} t_{1}^{-1} t_{4}\right)$ generate the other.

Placing $t_{3} t_{1}^{-1} t_{4}=x, t_{3} t_{1}^{-1}=y$ and $t_{3}=z$ gives a new presentation for the group,

$$
\left\langle x, y, z ; y \sim z, z=x^{-1} z\left(x^{-1} y\right)^{3} y^{-1} z x^{-1} y\right\rangle \text {. }
$$

The strategy now is to find a two component link with the above as fundamental group in such a way that $y$ and $z$ play the roles of meridian and longitude in one component (the trivial knot), while $x$ and $y z^{-1}\left(y^{-1} x\right)^{2} y z^{-1} y^{-1} x$ play the roles of meridian and longitude in the other (the trefoil knot). For ease in notation we name a knot by its meridian.

When the knot $y$ is filled in by a solid torus, an additional relation, $y=1$, has to be introduced into the link group: the resulting knot group is $\left\langle x, z ; z=x^{-1} z x^{-3} z x^{-1}\right\rangle$. Here, $x$ and $z^{-1} x^{2} z^{-1}$ generate a peripheral subgroup; the longitude of the original knot $y$ is represented by the element $x^{-1} z x^{-3} z x^{-1}$.

Using another transformation, $x=b$ and $z=b^{3} a b^{-1}$, we obtain the standard presentation $\langle a, b ; a b a=b a b\rangle$ of the left-handed trefoil knot with generators as given in the regular projection in Figure 2.

Notice that under this transformation the peripheral subgroup $\left\langle x, z^{-1} x^{2} z^{-1}\right\rangle$ is carried to the peripheral subgroup $\left\langle b, a b^{2} a\right\rangle$, so we see that the transformation respects the peripheral structure. The longitude of the knot $y$ is now represented by $\bar{b} \cdot b^{3} a b \cdot \bar{b}^{3} \cdot b^{3} a b \cdot \bar{b}=b^{2} a b a$.

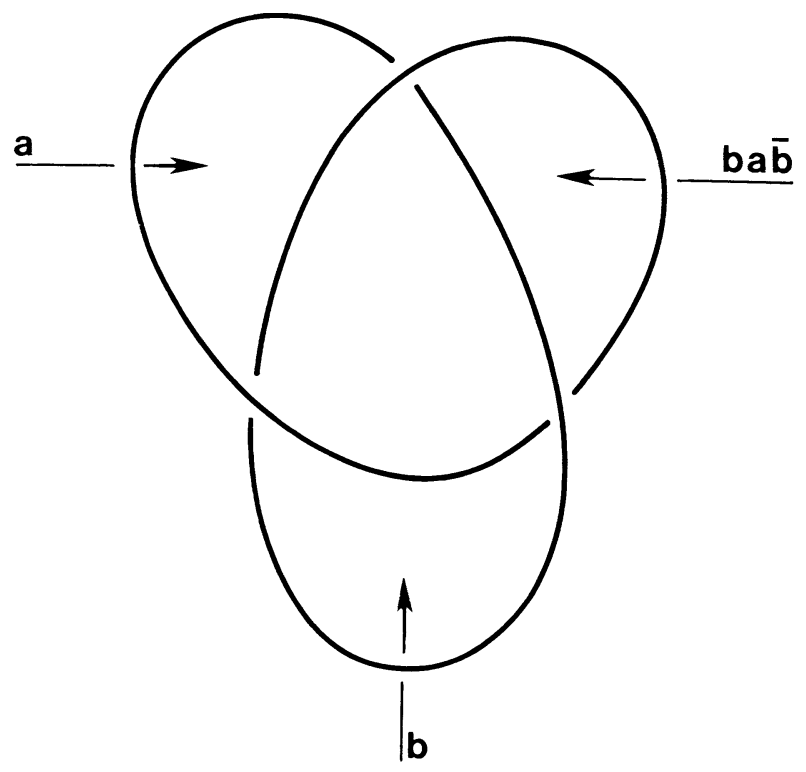

FIGURE 2 
We proceed to embed a circle into the complement of the knot in Figure 2 which represents $b^{2} a b a$ and thus obtain the link in Figure 3. Unfortunately, the choice of the embedding is not unique, as we could just as well have chosen any element in the free homotopy class of $b^{2} a b a$. We have chosen one which does not admit a self-crossing in its regular projection. It remains to show there is an isomorphism between the group of this link and the subgroup we are interested in. Then, since the isomorphism respects the peripheral structure, results of Riley [4], Waldhausen [9], show that this link complement is the required hyperbolic 3-manifold.

It can be seen from Figure 3 that the link group is generated by $a, b$ and $c$ together with two relations coming from brackets 5 and 10 .

\section{$\left.\begin{array}{ll}\text { 5. } & \bar{b} c \bar{a} \bar{b} c \bar{b} c \bar{b} \bar{c} b \bar{c} b a \bar{c} b a=1 \\ \text { 10. } & c \sim \bar{a} \bar{b} c \bar{a} \bar{b} c \bar{b}\end{array}\right\}$}

These two are equivalent to

$$
\bar{b} c \bar{a} \bar{b} c a \bar{c} b a \bar{c}=1, \quad c \sim \bar{a} \bar{b} c \bar{a} \bar{b} c \bar{b} .
$$

Placing $a=\bar{\beta} \alpha \gamma, b=\beta$ and $c=\gamma$

$$
\gamma=\alpha \beta \bar{\alpha}^{2} \beta, \quad \gamma \sim \bar{\alpha}^{2} \gamma \bar{\beta}, \quad \text { or } \alpha \beta \bar{\alpha}^{2} \beta \sim \bar{\alpha} \beta \bar{\alpha}^{2} .
$$

Finally, with $\alpha=t_{1} \bar{t}_{4}$ and $\beta=\left(t_{1} \bar{t}_{4}\right) \bar{t}_{1}\left(t_{1} \bar{t}_{4}\right)^{2}, \bar{t}_{4} t_{1} \bar{t}_{4}^{3} t_{1} \bar{t}_{4} \sim t_{1}$.

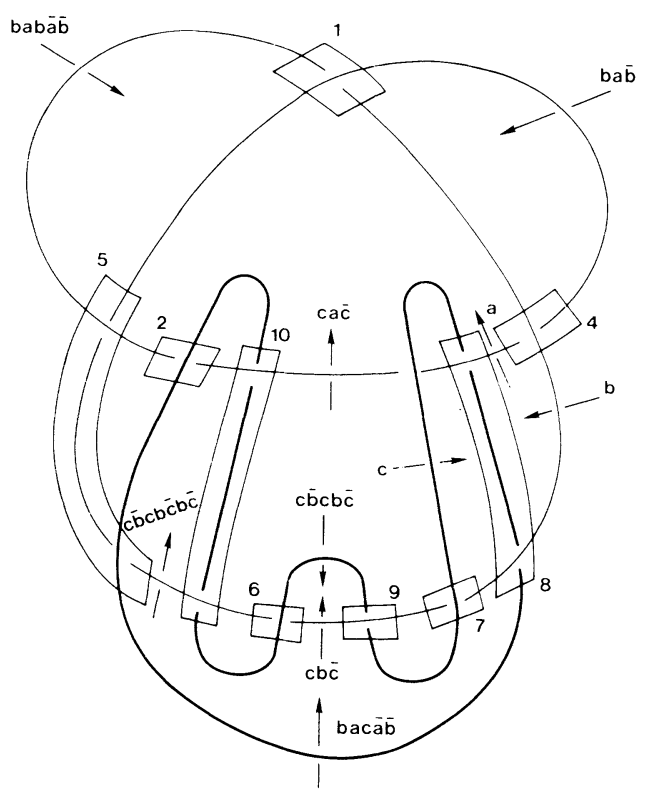

Figure 3

7. Subgroups of index 12. There are 24 representations of Pic into $S_{12}$ of the type discussed in $\$ 4$, which were produced by a computer. Those representations which are conjugate in $S_{12}$ produce isomorphic subgroups. We find in this way that the representations are conjugate to just two different ones; hence there are just two subgroups, which it turns out, are not isomorphic. Actually, it is not hard to show by hand there are just two inequivalent representations. 
One of these was studied in detail in $§ 6$, and the associated hyperbolic 3-manifold is the complement in $S^{3}$ of the link drawn in Figure 3. We denote this link by $W g$.

The other subgroup is isomorphic to the Whitehead link $W d$. The hyperbolic structure for $S^{3}-W d$ is now well known. For completeness, we give a representation for the group in $S_{12}$, a presentation arising from a corresponding $c$-cress, and a picture of $W d$.

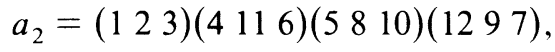

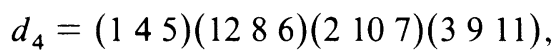

$$
\begin{aligned}
& b_{14}=\left(\begin{array}{ll}
4 & 1
\end{array}\right)(62)(95)(107)(113)(128) \text {, } \\
& c_{21}=\left(\begin{array}{ll}
6 & 5
\end{array}\right)\left(\begin{array}{l}
8 \\
1
\end{array}\right)(92)\left(\begin{array}{ll}
10 & 3
\end{array}\right)\left(\begin{array}{ll}
11 & 7
\end{array}\right)(124) \text {. }
\end{aligned}
$$

A presentation is $\left\langle u, v ; u \sim v u v^{-2} u v\right\rangle$, where

$$
u=b_{14}^{-1} a_{4}=\left(\begin{array}{ll}
1 & 0 \\
1 & 1
\end{array}\right), \quad v=c_{21}^{-1} a_{2} a_{5}=\left(\begin{array}{cc}
i & 1+i \\
-1 & -1
\end{array}\right)
$$

and $v u v^{-2} u v=\left(\begin{array}{cc}1 & 0 \\ 2 i & 1\end{array}\right)$. One peripheral subgroup is generated by $u$ and $v u v^{-2} u v$ with $\tau$-invariant $2 i$. From the symmetry of $W d$ (there is a homeomorphism of $S^{3}$ interchanging the two components), it follows that the $\tau$-invariant of the other component is also $2 i$. Since the $\tau$-invariants for $W g$ were each $i$, this shows that the groups $W d$ and $W g$ are not isomorphic. Another way to see these groups are not isomorphic is to compute the linking number; this is 0 for $W d$ and 5 for $W g$.

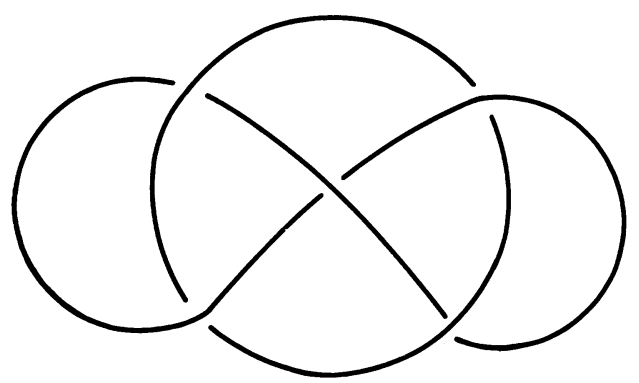

FIGURE 4

We note that the group of $W d$ is isomorphic to the group of the link with standard name $7_{8}^{2}$, so that $S^{3}-W d$ is homeomorphic to $S^{3}-7_{8}^{2}$. The links $W g$ and $7_{8}^{2}$ both consists of an unknot wound around a trefoil. $W g$ or an equivalent link does not appear in the standard table of links with 9 or fewer crossings. (See Rolfsen [6], where this notation for links is also explained.)

8. Subgroups of index greater than 12. The following result was communicated to us by D. Solitar: the index of a torsion-free subgroup $H$ of Pic is a multiple of 12 . For if $H$ is of finite index, there is a double-coset decomposition Pic $=\cup H x_{i} A$ with respect to $H$, where $A \cong A_{4}$ is the vertex group appearing in the polygonal product representation of Pic.

Now the number of distinct right cosets of $H$ in a fixed double coset $H x_{i} A$ is exactly $\left|A: x_{i}^{-1} H x_{i} \cap A\right|$. (To see this, note that $H x_{i} a=H x_{i} a^{\prime} \Leftrightarrow a^{\prime} a^{-1} \in x_{i}^{-1} H x_{i} \cap$ $\left.A \Leftrightarrow\left(x_{i}^{-1} H x_{i} \cap A\right) a=\left(x_{i}^{-1} H x_{i} \cap A\right) a^{\prime}.\right)$ 
However $H$ is torsion free and $A$ finite, so $x_{i}^{-1} H x_{i} \cap A=\{1\}$. Thus the number of distinct right cosets of $H$ in $H x_{i} A$ is exactly $|A|=12$. Hence $12 \|$ Pic: $H \mid$.

Karrass, Pietrowski and Solitar [2] have found that there is exactly one torsion-free normal subgroup of index 24 in Pic. This group turns out to be the group of the Borromean rings (isomorphic to case B.2 below).

Karrass, Pietrowski and Solitar [2] have also shown that the Picard group has no finitely generated normal subgroups of infinite index. This might lead to the suspicion that torsion-free subgroups of finite index are an infinite cyclic extension of a free group. However, this is not the case, as can be seen with the group $G$ in case A. 3 below.

$G$ has a presentation $\left\langle x, s, t ; t \sim \bar{s}^{2} \bar{x} s^{2} x, \bar{t} x t=\bar{s} x s x\right\rangle$. It is not hard to see that $G / G^{\prime \prime}$ has a presentation $\left\langle\left\langle x, s, t ; \bar{s} x s=x, \bar{t} x t=x^{2}\right\rangle\right\rangle$, where double brackets are used here to indicate that relations implying that commutators commute have been supressed. Consequently, $G^{\prime} / G^{\prime \prime}$ is the direct product of an infinitely generated free abelian group together with a copy of the dyadic rationals. It follows that $G^{\prime}$ is not a free group, and hence that $G$ is not an infinite cyclic extension of a free group.

An interesting sequence of examples with index $12 m$ comes from the cyclic coverings of $S^{3}-W g$. Since one component of $W g$ is unknotted, the cyclic covering spaces branched along this component are also links in $S^{3}$, their complements are hyperbolic, and the groups are subgroups of index $12 \mathrm{~m}$ in Pic. Furthermore, in each case, the link has two components, except when $m=5$ (there are 6 components in that case). One component is an unknot, the other is an $m$-fold cover of the trefoil. The hyperbolic volume of the complement is $12 \mathrm{~m}$ times the hyperbolic volume for Pic. The link is shown in Figure 5 for $m=2$.

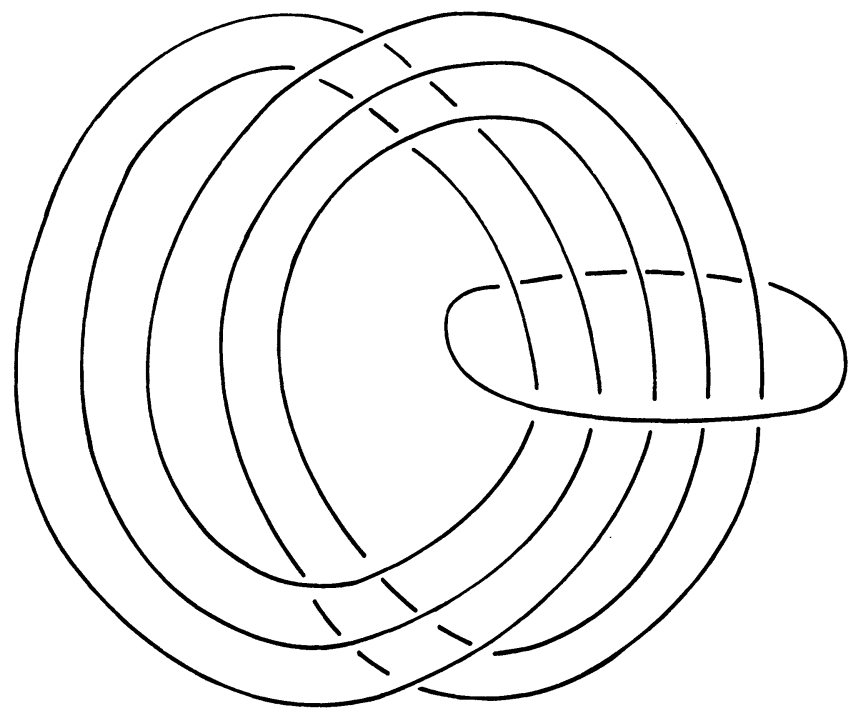

FIGURE 5 
9. Computing representations in $S_{n}$. From the previous section, the index of a torsion-free subgroup, $H$, is $n=12 \mathrm{~m}$. Now $a_{2}$ and $d_{4}$ must each be a product of $n / 3$ disjoint 3-cycles. Our notation is chosen so that $a_{2}$ takes 1 to $2, a_{3}=a_{2}^{2}$ takes 1 to 3 , $d_{4}$ takes 1 to 4 , and $d_{5}=d_{4}^{2}$ takes 1 to 5 (see $\S 4$ ). It can easily be shown that there are $m$ elements, $g_{1}=$ identity of Pic, $g_{2}, \ldots, g_{m}$ in Pic such that $\left\{H g_{i} a_{j}: i=\right.$ $1,2, \ldots, m ; j=1,2, \ldots, 12\}$ is the set of right cosets of $H$ in Pic. This implies that any regular representation of Pic in $S_{n}$ is conjugate in $S_{n}$ to a representation where

$$
\begin{aligned}
a_{2}= & \prod_{0 \leqslant k \leqslant m-1}(1+12 k 2+12 k 3+12 k)(4+12 k 11+12 k 6+12 k) \\
& \cdot(5+12 k 8+12 k 10+12 k)(12+12 k 9+12 k 7+12 k), \\
a_{4}= & d_{4}=\prod_{0 \leqslant k \leqslant m-1}(1+12 k 4+12 k 5+12 k)(12+12 k 8+12 k 6+12 k) \\
& \cdot(2+12 k 10+12 k 7+12 k)(3+12 k 9+12 k 11+12 k),
\end{aligned}
$$

In other words, we can produce 3-cycles in blocks of four by adding a multiple of 12 to each integer in the first four 3-cycles of $a_{2}$ and $d_{4}$. Since we are only interested in finding torsion-free subgroups of index $n$ up to isomorphism, $a_{2}$ and $d_{4}$ may be always chosen to be the fixed elements above.

Each of $b_{14}$ and $c_{21}$ are a product of $n / 2$ disjoint 2-cycles. As $n$ becomes large this gives an enormous number of candidates for regular, transitive representations. The strategy is to use regularity and the group relations to reduce the number of candidates as much as possible. For $n=12$, a total of 24 representations were produced. For $n=24$, the total number of representations is large (>2000). We then eliminated chunks of cases which differ by an automorphism of either the Picard group or of $S_{24}$. With these reductions our computer program listed about 300 representations. Many of these were still equivalent under automorphisms. The final elimination of isomorphic subgroups was done by using the group presentations, the Ford domains, and the $\tau$-invariants.

The following example illustrates our algorithm for producing representations. When $n=24$, we may take

$$
\begin{aligned}
& a_{2}=\left(\begin{array}{lll}
12 & 3
\end{array}\right)\left(\begin{array}{l}
4 \\
11
\end{array} 6\right)(5810)(1297)(131415)(162318)(172022)(242119) \text {, } \\
& d_{4}=\left(\begin{array}{ll}
145 \\
1
\end{array}\right)(1286)(2107)(3911)(131617)(242018)(142219)(152123) \text {. }
\end{aligned}
$$

For $b_{14}$, start with the 2-cycle (1 13$)$. In order to satisfy $\bar{b}_{14} b_{2} b_{14}=\bar{b}_{2}$ we must also then have $(215)$ and $(314)$. Now, a choice is necessary for the next 2-cycle and all the possibilities must be checked. Try (45); in order to satisfy $\bar{b}_{14} b_{2} b_{14}=\bar{b}_{2}$ we must also then have (11 10) and (6 8). Continue in this way to complete $b_{14}$, if possible. For each $b_{14}$ constructed, there are many candidates for $c_{21}$. To continue our example, take

$$
b_{14}=\left(\begin{array}{ll}
1 & 13
\end{array}\right)(215)(314)(417)\left(\begin{array}{ll}
11 & 22
\end{array}\right)(6 \quad 20)(5 \text { 16)(8 18)(10 23)(7 21)(12 24)(9 19) }
$$

and begin with $\left(\begin{array}{ll}1 & 2\end{array}\right)$ as a "seed" for $c_{21}$. The relation $\bar{d}_{4} b_{14} d_{4}=\bar{b}_{14}$ produces the 2-cycles (4 7) and (5 10). Now, the relations at vertex $C$ can be used to produce a 
new "seed" to continue $c_{21}$; for example, the relation $\bar{c}_{14} c_{21} c_{14}=\bar{c}_{21}$ produces the 2-cycles (17 21) and (16 23). At this point, there are two possibilities: in the one case, one of the old 2-cycle and the relations at vertex $C$ produce a new 2-cycle; in the other, none of the old 2-cycles and the relations at vertex $C$ produces a new 2-cycle. In the second case, many possible 2-cycles must be generated and checked. In either case, the relations of $C$ and $D$ are used as described to complete $c_{21}$, if possible.

10. Subgroups of index 24. Up to isomorphism, there are 17 different torsion-free subgroups of index 24. Seven of these are link groups, where the link is in $S^{3}$, and has two, three, or four components. The other manifolds are the complements of links in 3-manifolds such as $S^{1} \times S^{2}$, lens spaces, mapping tori and homology 3 -spheres. Four of the manifolds have only one boundary torus; that is, the group has only one conjugacy class of peripheral subgroups. No group is the group of a knot in $S^{3}$.

We list these manifolds and groups in classes based on their first homology, number of boundary tori, and/or the shape of the Ford domain.

(A) Link groups with $H_{1}=2 \mathbf{Z}$.

(B) Link groups with $H_{1}=3 \mathbf{Z}$.

(C) Link groups with $H_{1}=4 \mathrm{Z}$.

(D) Link in a homology 3-sphere with $H_{1}=2 \mathbf{Z}$.

(E) One boundary torus and $H_{1}=2 \mathbf{Z}$.

(F) One boundary torus and $H_{1}=\mathbf{Z} \oplus \mathbf{Z}_{5}$.

(G) One boundary torus and $H_{1}=\mathbf{Z} \oplus 2 \mathbf{Z}_{3}$.

(H) Two boundary tori and $H_{1}=2 \mathbf{Z} \oplus \mathbf{Z}_{3}$.

(I) Two boundary tori and $H_{1}=2 \mathbf{Z} \oplus \mathbf{Z}_{4}$.

(J) Two boundary tori and $H_{1}=2 \mathbf{Z} \oplus \mathbf{Z}_{5}$.

A few of these manifolds will be double covers of either $S^{3}-W g$ or $S^{3}-W d$. Given any 2-generator group generated by $\{x, y\}$, there are three possible subgroups of index 2:
(a) $\operatorname{sbgp}\left\langle y^{2}, x, \bar{y} x y\right\rangle$,
(b) $\operatorname{sbgp}\left\langle x^{2}, y, \bar{x} y x\right\rangle$
and
(c) $\operatorname{sbgp}\left\langle\bar{x} y, y \bar{x}, y^{2}\right\rangle$.

The usual Reidemeister-Schreier rewriting process can be used to provide presentations.

In the case of $\pi_{1}\left(S^{3}-W g\right)=\left\langle x, y ; x \sim \bar{y} x y^{3} x \bar{y}\right\rangle$, there is an outer automorphism $x \rightarrow \bar{y} x \bar{y}^{3} x \bar{y}, y \rightarrow \bar{y} x \bar{y}$ which induces an isomorphism between the subgroups in (a) and (b). It is not hard to see from the presentations derived that case (a) and case (c), respectively, give the groups associated with the double cover branched along the unknot given in A.l below, and branched along the trefoil given in H.l below.

Similarly for $\pi_{1}\left(S^{3}-W d\right)=\left\langle x, y ; x \sim \bar{y} x y^{2} x \bar{y}\right\rangle$, there is an outer automorphism $x \rightarrow y x, y \rightarrow \bar{y}$ which induces an isomorphism between the subgroups in (a) and (c). It is not hard to see from the presentations derived that case (a) and case (b), respectively, give the groups associated with the double cover branched along one component given in B.1 below, and branched along both components given in I.2 below. 
In all cases, the representations for $a_{2}$ and $d_{4}$ in $S_{24}$ are fixed as given in $\S 9$. To identify and describe the manifolds we use a mixture of working with the group presentations, and the methods and terminology of surgery on 3-manifolds. The first step is usually to find the peripheral subgroups; this is done either algebraically, or from the cycles of ideal vertices of the Ford domain. Then, filling in one or more boundary tori with solid tori may determine part of the manifold. In most cases, we present the final results without describing the method of getting there.

In interpreting the Ford domains, recall that transformations which pair hemispheres can be broken up into an inversion in one hemisphere, reflection in a plane through its centre and perpendicular to $\mathbf{C}$, rotation about the centre, and translation to the other hemisphere. (It is possible that the rotation is the identity mapping.)

The pairing of hemispheres is usually indicated in the diagrams by capital letters such as $A, B, C, D$. These letters correspond also to words in the generators of the group. For the sake of brevity, the words are not given. In some cases, there is a unique way for an orientation-preserving isometry, say $A$, to accomplish the indicated identification. In other cases we indicate that $A$ contains a reflection in a horizontal plane (perpendicular to $\mathbf{C}$, parallel to the $x$-axis, through the center of the hemisphere) or a vertical plane (perpendicular to $\mathbf{C}$, parallel to the $y$-axis, through the center of the hemisphere); or one of these reflections and a rotation of the hemisphere.

For example, in Figure 1 , the isometry $t_{1}$ consists of inversion, reflection in a vertical plane, and translation by -2 . The isometry $t_{3}$ consists of inversion, reflections in a horizontal plane, and translation by $2 i$. In Figure $6, A$ and $D$ contain reflections in a horizontal plane and no rotation, while $B$ and $C$ contain reflections in a vertical plane and no rotation. In all cases one must think of inversion, then reflection, then a possible rotation and a translation.

The generators $\left(\begin{array}{ll}1 & w_{1} \\ 0 & 1\end{array}\right)$ and $\left(\begin{array}{ll}1 & w_{2} \\ 0 & 1\end{array}\right)$ of the stabilizer of $\infty$ are indicated by giving $w_{1}$ and $w_{2}$. The group presentations given are those for the matrix groups.

A.1. Double cover of $S^{3}-W g$ branched along the unknot.

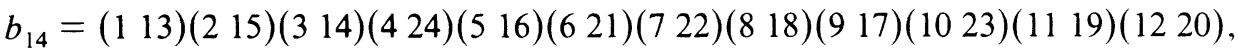

$$
\begin{aligned}
& c_{21}=\left(\begin{array}{ll}
1 & 2
\end{array}\right)(312)(47)(510)(69)(811)(1315)(1420)(1623)(1721)(1819)(2224) \text {. }
\end{aligned}
$$

A $c$-cress is given in Table 3. In producing a presentation, and reversing the order of letters we obtain a presentation as a matrix group $\left\langle u, v, w ; v \sim w^{2} u v \bar{w} u\right.$, $u \bar{w} u=\bar{v} w u v>$ where

$$
u=\left(\begin{array}{cc}
-2 i & i \\
-2+i & 1
\end{array}\right), \quad v=\left(\begin{array}{cc}
1 & -i \\
0 & 1
\end{array}\right), \quad w=\left(\begin{array}{cc}
2+i & 1-i \\
-1-2 i & -1
\end{array}\right)
$$

A Ford domain is given in Figure 6 below. One peripheral subgroup is $\left\{v, w^{2} u v \bar{w} u\right\}$ with meridian $v$, and the other is $\left\{u v, \bar{w}^{2} v w v\right\}$ with meridian $\bar{w}^{2} v w v$. Both components are unknotted and have $\tau$-invariant $2 i$. The link is given in Figure 5. 
TABLE 3

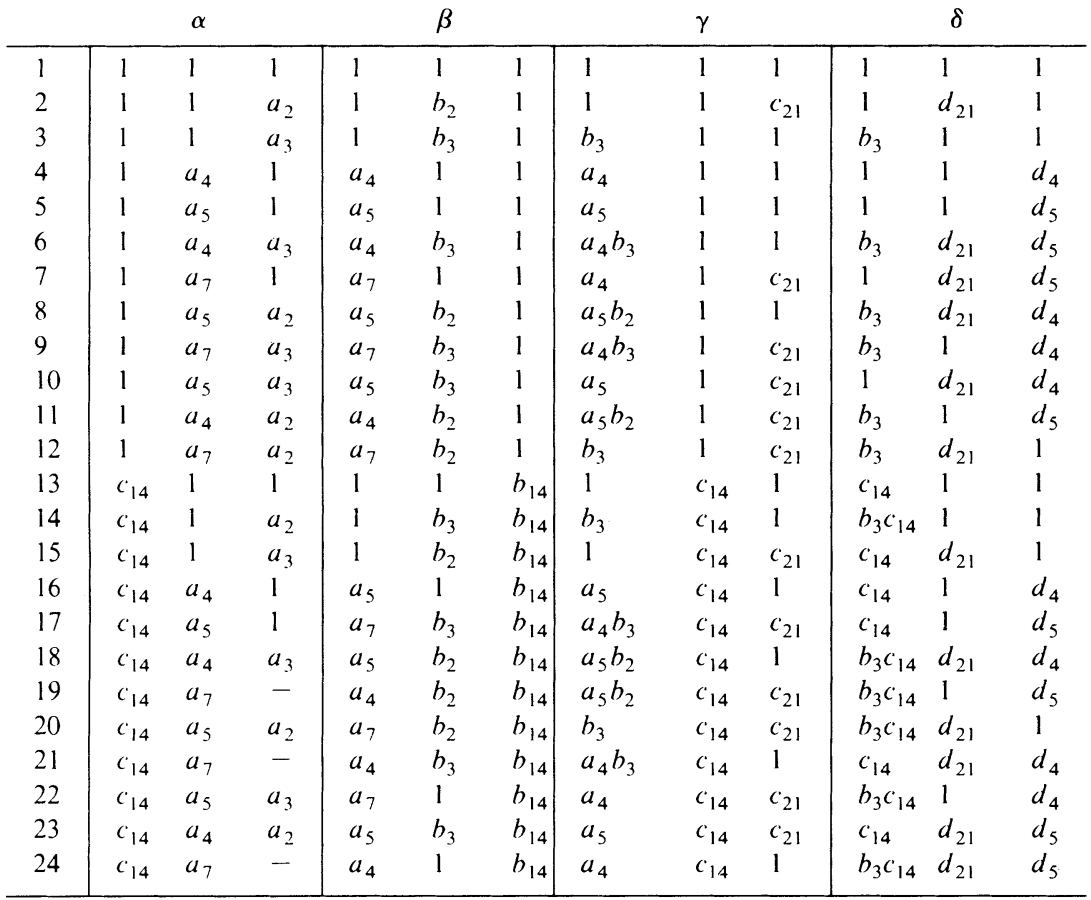

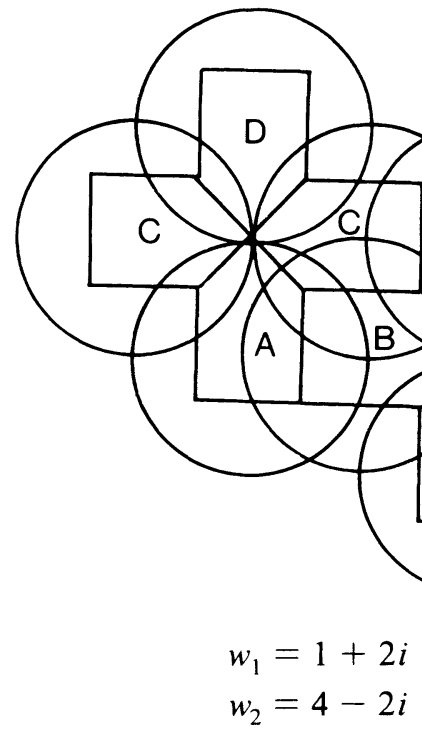

Figure 6

A.2. Unknot and the square knot.

$b_{14}=\left(\begin{array}{ll}1 & 13\end{array}\right)(215)(314)(417)(516)(620)(721)(818)(9$ 19)(10 23)(11 22)(12 24), $c_{21}=\left(\begin{array}{ll}1 & 2\end{array}\right)(36)(47)(510)(8$ 9)(11 12)(13 15)(14 20)(16 23)(17 21)(18 19)(22 24). 
A presentation is $\langle u, v, z ; u \sim \bar{v} u z \bar{u} v z, u z \bar{u}=\bar{v} z v z\rangle$ where

$$
u=\left(\begin{array}{ll}
1 & 0 \\
1 & 1
\end{array}\right), \quad v=\left(\begin{array}{cc}
-2-i & -2+i \\
1+i & 1
\end{array}\right), \quad u z \bar{u}=\left(\begin{array}{cc}
1 & 2 i \\
0 & 1
\end{array}\right) .
$$

Then

$$
\bar{v} u z \bar{u} v z=\left(\begin{array}{cc}
1 & 0 \\
-2 i & 1
\end{array}\right) \text { and } u v^{2} u \bar{v}=\left(\begin{array}{ll}
1 & 5 \\
0 & 1
\end{array}\right)
$$

The peripheral subgroup associated with the unknot is $\{u, \bar{v} u z \bar{u} v z\}$ with meridian $u$ and $\tau$-invariant $5 i / 2$; the peripheral subgroup associated with the square knot is $\left\{u z \bar{u}, u v^{2} u v\right\}$ with meridian $u v^{2} u v$ and $\tau$-invariant $2 i$. A Ford domain is given in Figure 7 and the link in Figure 8.

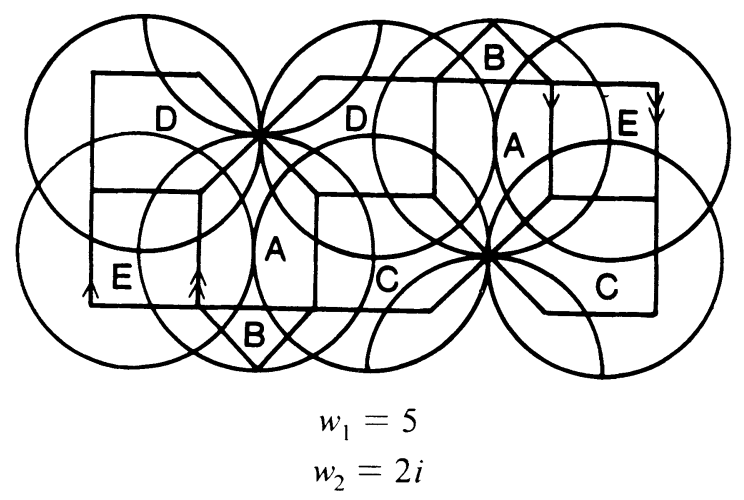

Figure 7

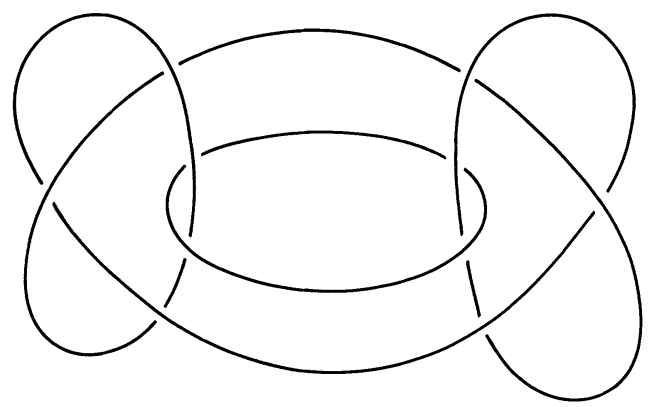

Figure 8

A.3. Unknot and the knot $6_{1}$.

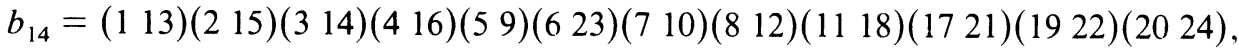

$$
\begin{aligned}
& c_{21}=\left(\begin{array}{ll}
1 & 16
\end{array}\right)(218)(323)(413)(5 \text { 17)(6 14)(7 24)(8 22)(9 21)(10 20)(11 15)(12 19). }
\end{aligned}
$$

A presentation is $\left\langle u, v, w ; u \sim v^{2} \bar{w} \bar{v}^{2} w, \bar{v} w u \bar{w} v=u w \bar{v}\right\rangle$ where

$$
u=\left(\begin{array}{cc}
1 & 0 \\
-1 & 1
\end{array}\right), \quad v=\left(\begin{array}{cc}
-2+2 i & -3 \\
1-2 i & 2-i
\end{array}\right), \quad w=\left(\begin{array}{cc}
-2-2 i & 1-2 i \\
1 & i
\end{array}\right) \text {. }
$$


Also,

$$
v^{2} \bar{w} \bar{v}^{2} w=\left(\begin{array}{cc}
1 & 0 \\
-2 i & 1
\end{array}\right), \quad v^{2} \bar{w}^{2}=\left(\begin{array}{cc}
1 & 2 i \\
0 & 1
\end{array}\right), \quad w \bar{u} \bar{w} \dot{v} u=\left(\begin{array}{cc}
1 & -5+i \\
0 & 1
\end{array}\right)
$$

Then the peripheral subgroup associated with the unknot is $\left\{u, v^{2} \bar{w} \bar{v}^{2} w\right\}$ with meridian $u$ and $\tau$-invariant $\frac{1}{2}+\frac{5}{2} i$; the peripheral subgroup associated with the knot $6_{1}$ is $\left\{v^{2} \bar{w}^{2}, w \bar{u} \bar{w} \bar{v} u\right\}$ with meridian $w \bar{u} \bar{w} \bar{v} u$ and $\tau$-invariant $2 i$. The Ford domain is given in Figure 7 with two changes in the face-pairing transformations; the identification of the hemispheres labelled $A, B$ is changed by a $180^{\circ}$ rotation, and the translation by 5 units is replaced by $5+i$. In fact, the same Ford domain could be used for each of the groups A.1, A.2, A.3. The link for A.3 is given in Figure 9.

The next four groups have Ford domains which can be described as two copies of a Ford domain for $W d$; that is, two regular ideal hyperbolic octahedra. The groups B.1, B.2, and C have appeared with more details in Wielenberg [10].

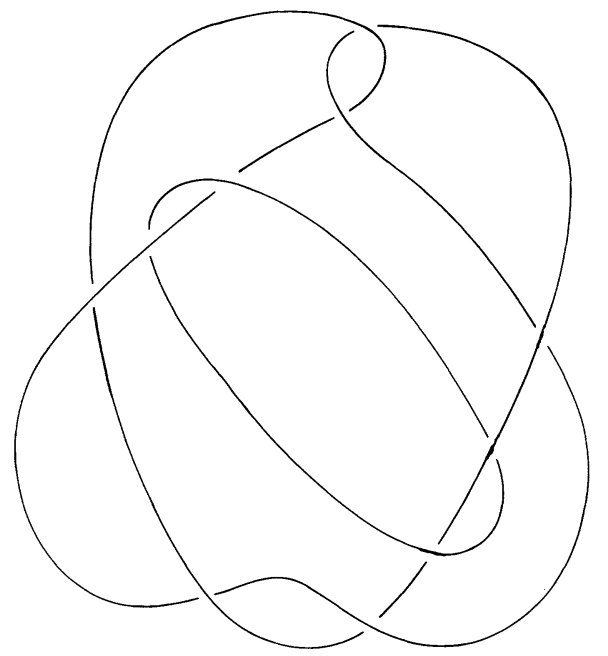

FIGURE 9

B.1. Double cover of $S^{3}-W d$ branched along one component $=$ the link $8_{9}^{3}$.

$b_{14}=\left(\begin{array}{ll}1 & 13\end{array}\right)(215)(314)(421)\left(\begin{array}{l}5 \\ 16\end{array}\right)(6$ 19)(7 17)(8 18)(9 20)(10 23)(11 24)(12 22), $c_{21}=\left(\begin{array}{ll}1 & 2\end{array}\right)(38)(47)(510)(611)(912)(1315)(14$ 18)(16 23)(17 21)(19 24)(20 22).

A presentation is $\langle u, v, w ; v \sim u \bar{w} \bar{u} \bar{w}, w \sim \bar{v} \bar{u} \bar{v} u\rangle$. The $\tau$-invariants for the peripheral subgroups are $i, 2 i$ and $2 i$.

B.2. The Borromean rings $=$ the link $6_{2}^{3}$.

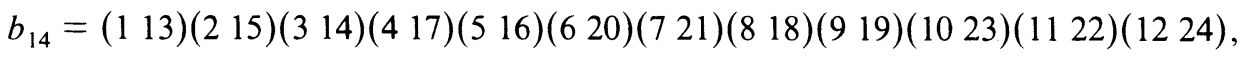
$c_{21}=\left(\begin{array}{l}12 \\ 2\end{array}\right)\left(\begin{array}{l}3 \\ 8\end{array}\right)(47)(510)(611)(9$ 12)(13 15)(14 18)(16 23)(17 21)(19 24)(20 22).

A presentation is $\langle u, v, w ; v \sim u w \bar{u} \bar{w}, w \sim v \bar{u} \bar{v} u\rangle$. The $\tau$-invariant for each peripheral subgroup is $2 i$. 
B.3. Three unknots.

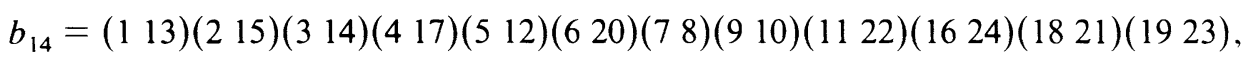

$c_{21}=\left(\begin{array}{ll}1 & 22\end{array}\right)(2$ 20)(3 17)(4 14)(5 19)(6 15)(7 18)(8 21)(9 16)(10 24)(11 13)(12 23).

A presentation is $\langle u, v, w, z ; u \sim v, w \sim z, z u \bar{v} z u \bar{w} v \bar{w} u=1\rangle$ where

$$
\begin{gathered}
u=\left(\begin{array}{ll}
1 & 2 \\
0 & 1
\end{array}\right), \quad v=\left(\begin{array}{cc}
1 & 1+2 i \\
0 & 1
\end{array}\right), \\
w=\left(\begin{array}{cc}
1 & 0 \\
1-i & 1
\end{array}\right), \quad z=\left(\begin{array}{cc}
1 & 0 \\
-1-i & 1
\end{array}\right) .
\end{gathered}
$$

The three peripheral subgroups are $\{u, v\},\{w, z\}$ and $\left\{v w z v u, v(z v u)^{2} w v\right\}$ with $\tau$-invariants $\frac{1}{2}+i, i$ and $\frac{1}{2}+\frac{3}{2} i$, respectively. The link is given in Figure 11 and a Ford domain in Figure 10.

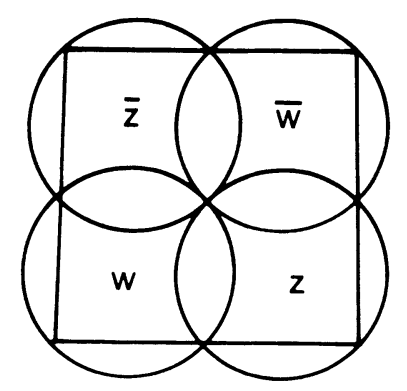

FIGURE 10

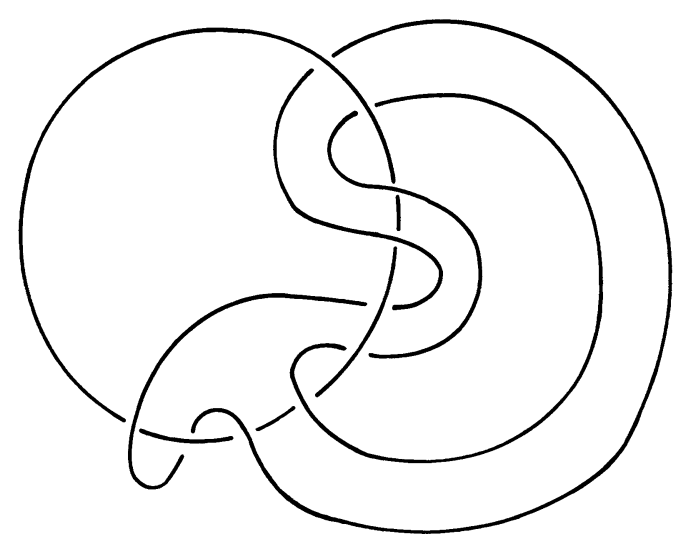

FIGURE 11

C. The link $8_{2}^{4}$.

$b_{14}=\left(\begin{array}{ll}1 & 13\end{array}\right)(215)(314)(417)(516)(620)(721)(818)(919)(1023)(1122)(1224)$,

$c_{21}=\left(\begin{array}{ll}1 & 22\end{array}\right)(2$ 20)(3 17)(4 14)(5 19)(6 15)(7 18)(8 21)(9 16)(10 24)(11 13)(12 23).

A presentation is $\langle u, r, w, z ; u \sim r, w \sim z, r \bar{w} u z \bar{r} w \bar{u} \bar{z}=1\rangle$. The matrices for $u, w$, $z$ are the same as in B.3, and $r=\left(\begin{array}{ll}1 & 2 i \\ 0 & 1\end{array}\right)$. The Ford domain is given in Figure 10. The 
peripheral subgroups are $\{u, r\},\{w, z\},\{\bar{r} \bar{u} w, \bar{u} \bar{z} r\}$ and $\{w \bar{u} \bar{z}, r \bar{z} \bar{w}\}$, each with $\tau$-invariant $i$.

D. A link in a homology 3-sphere.

$$
\begin{aligned}
& b_{14}=\left(\begin{array}{ll}
1 & 13
\end{array}\right)(215)(314)(421)(57)(619)(89)(1012)(1124)(1620)(1723)(1822), \\
& c_{21}=\left(\begin{array}{ll}
1 & 9
\end{array}\right)(218)(34)(511)(617)(724)(813)(1020)(1216)(1421)(1522)(1923) .
\end{aligned}
$$

A presentation is $\left\langle x, y, z ; x \sim y, z x \bar{z}^{2} x \bar{z}^{2} x z=y \bar{z} \bar{x} y \bar{z} y\right\rangle$ where

$$
x=\left(\begin{array}{cc}
1 & -1-2 i \\
0 & 1
\end{array}\right), \quad y=\left(\begin{array}{cc}
1 & 2-2 i \\
0 & 1
\end{array}\right), \quad z=\left(\begin{array}{cc}
-1 & 1+i \\
-1 & i
\end{array}\right) .
$$

One peripheral subgroup is $\{x, y\}$ with meridian $x \bar{y}$ and $\tau$-invariant $\frac{2}{5}+\frac{6}{5} i$; the other is $\left\{\bar{z} y \bar{z} y \bar{z}^{2}, z^{2} \bar{x} \bar{z} y \bar{z} \bar{x} z\right\}$ with meridian $z^{2} \bar{x} \bar{z} y \bar{z} \bar{x} z$ and $\tau$-invariant $\frac{2}{5}+\frac{6}{5} i$. The Ford domain is given in Figure 12, and the manifold in Figure 13. The manifold is the complement of the two component link drawn in the Poincare homology sphere (which is given here as a +1 framed surgery on the right-handed trefoil). We have not been able to show that this is not the group of a link in $S^{3}$.

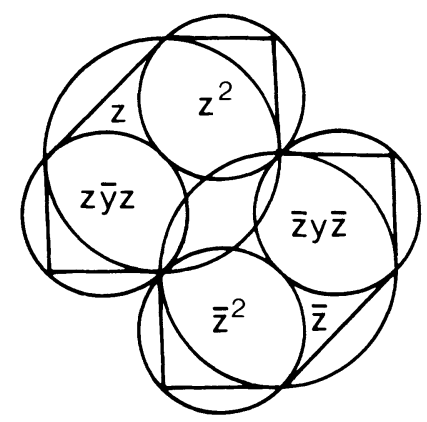

FiguRE 12

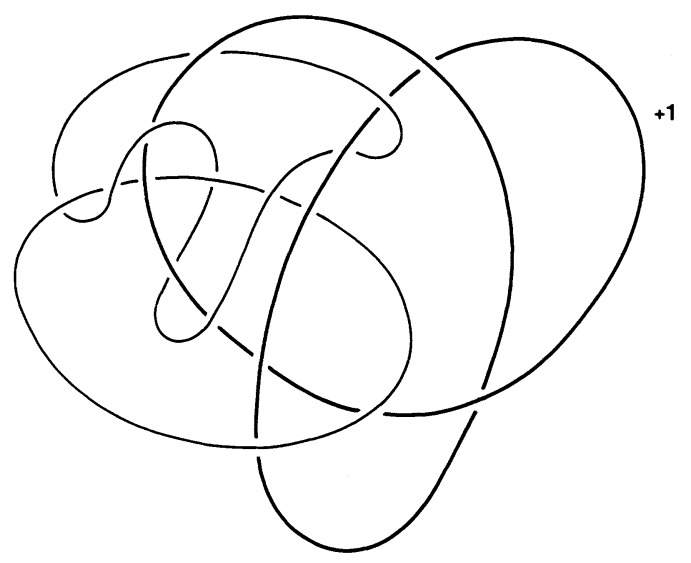

FIGURE 13 
E.1. A knot in a closed manifold which is doubly covered by 0 -surgery on the connected sum of two figure 8 knots.

$b_{14}=\left(\begin{array}{ll}1 & 13\end{array}\right)(2$ 15)(3 14)(4 17)(5 16)(6 20)(7 21)(8 18)(9 19)(10 23)(11 22)(12 24), $c_{21}=\left(\begin{array}{ll}1 & 14\end{array}\right)(224)(313)(4$ 19)(5 22)(6 21)(7 20)(8 23)(9 17)(10 18)(11 16)(12 15).

A presentation is $\left\langle u, v, w ; \bar{v} u v=\bar{w}^{2} u w^{2} \bar{u}, \bar{v} \bar{w}^{2} u w^{2} v=\bar{w}^{2} u w^{2} \bar{w} u w\right\rangle$ where

$$
u=\left(\begin{array}{cc}
1 & -2 \\
0 & 1
\end{array}\right), \quad v=\left(\begin{array}{cc}
1+2 i & 2+i \\
1 & 1-i
\end{array}\right), \quad w=\left(\begin{array}{cc}
-i & -1 \\
1 & 0
\end{array}\right) .
$$

The peripheral subgroup is $\left\{u, v u v u w \bar{u} \bar{w}^{2}\right\}$ with $\tau$-invariant $3 i$. The manifold is a knot in a closed manifold which is doubly covered by the manifold obtained as 0 -surgery on the connected sum of two figure 8 knots. It is given in Figure 15, and the Ford domain in Figure 14.

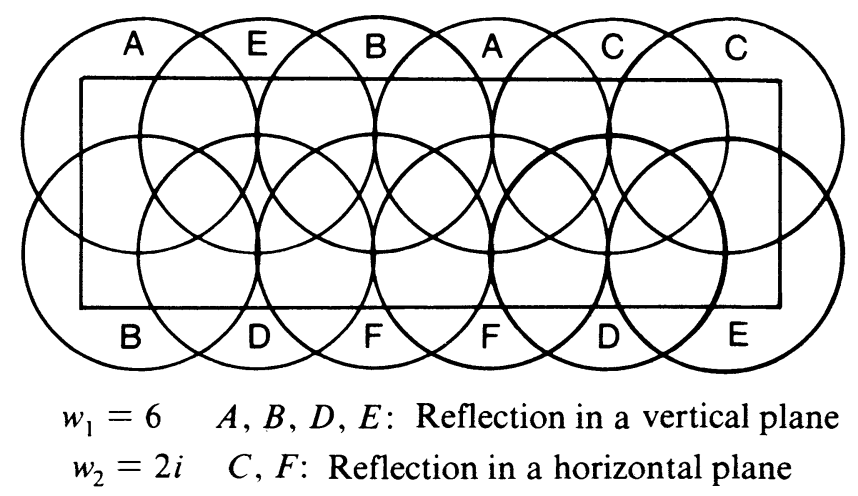

FIGURE 14

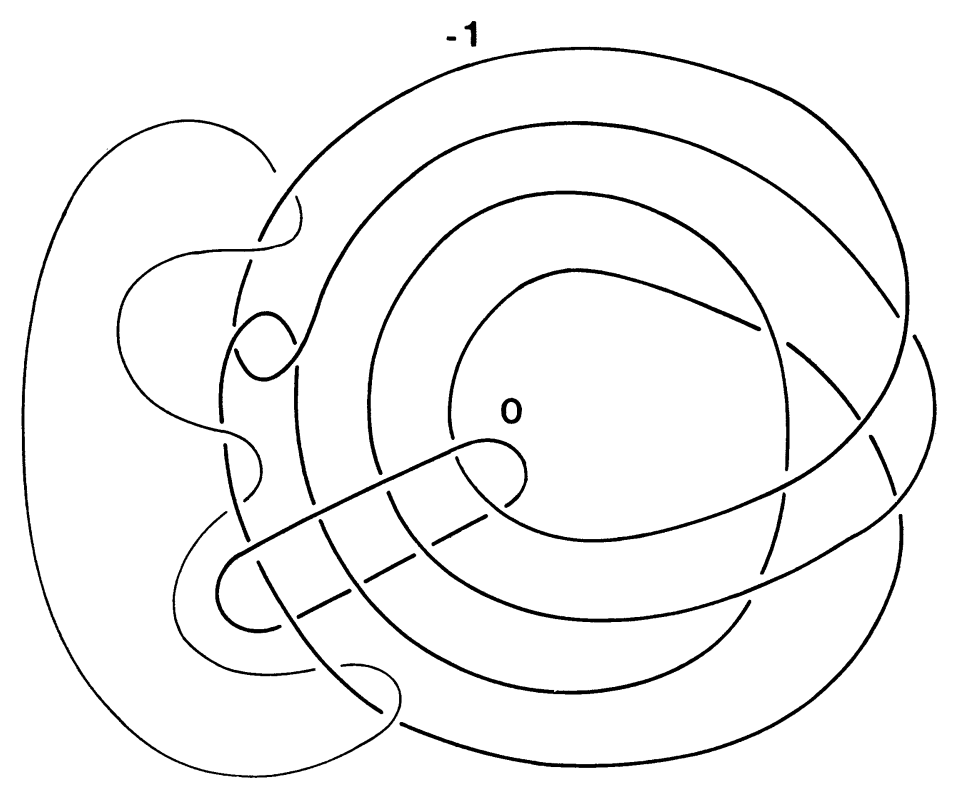

FigURE 15 
E.2. A knot in the manifold obtained as 0 -surgery on $10_{153}$.

$b_{14}=\left(\begin{array}{ll}1 & 13\end{array}\right)(215)(314)(416)(57)\left(\begin{array}{l}6 \\ 2\end{array}\right)(8$ 9)(10 12)(11 18)(17 24)(19 20)(21 22),

$c_{21}=\left(\begin{array}{ll}1 & 16\end{array}\right)(2$ 18)(3 23)(4 13)(5 17)(6 14)(7 24)(8 22)(9 21)(10 20)(11 15)(12 19).

A presentation is $\left\langle u, v, w ; \bar{w}^{2} \bar{u} v w^{2}=\bar{u}^{2} v^{2}, \bar{w} \bar{u} v w=\bar{v}^{3} u^{3}\right\rangle$ where

$$
u=\left(\begin{array}{cc}
-2 i & 2-i \\
i & i-1
\end{array}\right), \quad v=\left(\begin{array}{cc}
-2 i & -2-i \\
i & 1+i
\end{array}\right), \quad w=\left(\begin{array}{cc}
-i & -i \\
-i & 0
\end{array}\right) .
$$

The peripheral subgroup is $\left\{\bar{u} v, w^{2} v w \bar{u}^{2}\right\}$ with $\tau=$ invariant $\frac{1}{2}+3 i$. The manifold is obtained as a knot in the closed manifold which is obtained by 0 -surgery on $10_{153}$. The manifold is given in Figure 17 and the Ford domain in Figure 16.

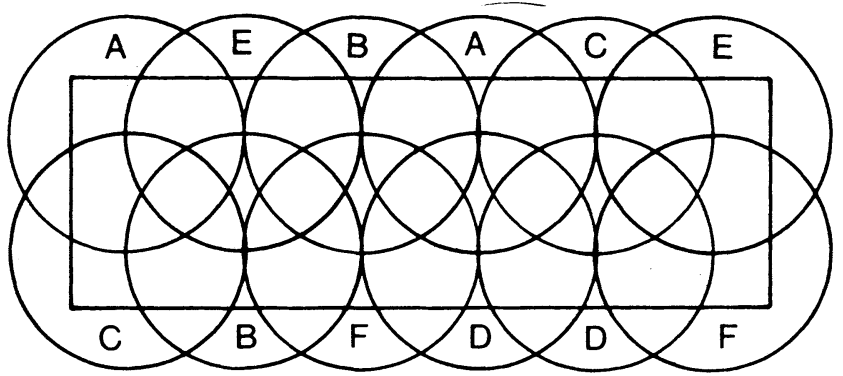

$w_{1}=6+i \quad A, B, C, D:$ Reflection in a horizontal plane $w_{2}=2 i \quad E, F:$ Reflection in a vertical plane

FIGURE 16

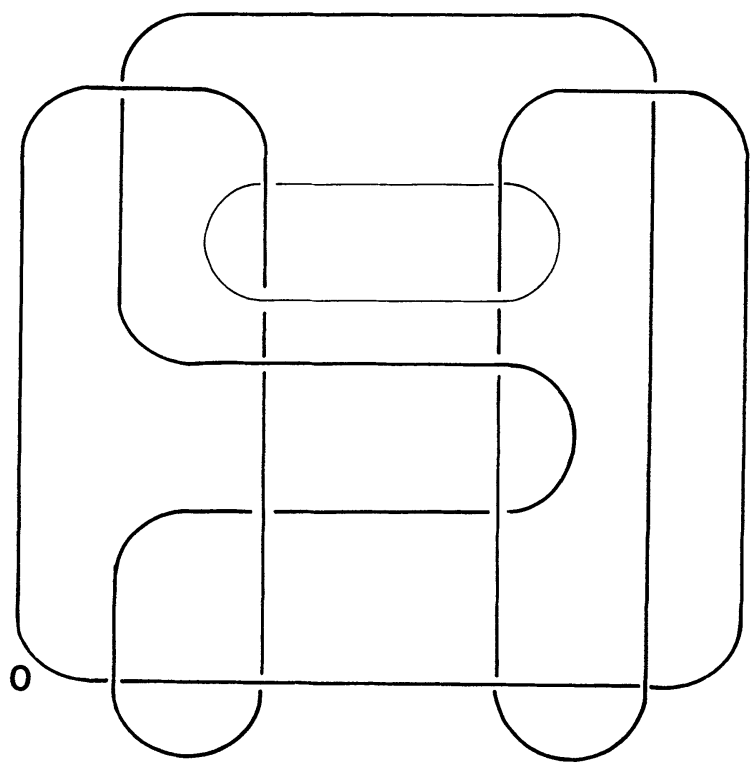

FIGURE 17

F.1. Knot in 5-surgery manifold on figure-eight knot.

$b_{14}=\left(\begin{array}{ll}1 & 11\end{array}\right)(24)(36)(512)(78)(910)(1318)(1423)(1516)(1719)(2021)(2224)$,

$c_{21}=\left(\begin{array}{ll}1 & 18\end{array}\right)(221)(317)(420)(524)(6 \quad 19)(723)(8$ 14)(9 16)(10 15)(11 13)(12 22). 
A presentation is

$$
\left\langle x, y, c ; \bar{x} y \bar{x}^{2} y c \bar{x}=y c \bar{x} y^{3} c \bar{x} y, \bar{y} x \bar{y}^{2} x \bar{c} \bar{y}=x \bar{c} \bar{y} x^{3} \bar{c} \bar{y} x\right\rangle
$$

where

$$
x=\left(\begin{array}{cc}
i & -1+i \\
-i & 1-2 i
\end{array}\right), \quad y=\left(\begin{array}{cc}
0 & i \\
i & 1-i
\end{array}\right), \quad c=\left(\begin{array}{cc}
1 & 1+4 i \\
0 & 1
\end{array}\right) .
$$

The peripheral subgroup is $\left\{c, \bar{x}^{2} y c \bar{x} y^{2}\right\}$ with meridian $c$ and $\tau$-invariant $\frac{1}{3}+\frac{4}{3} i$. The Ford domain is given in Figure 18. The manifold is given in Figure 19 showing the (meridinal) generators for the fundamental group.

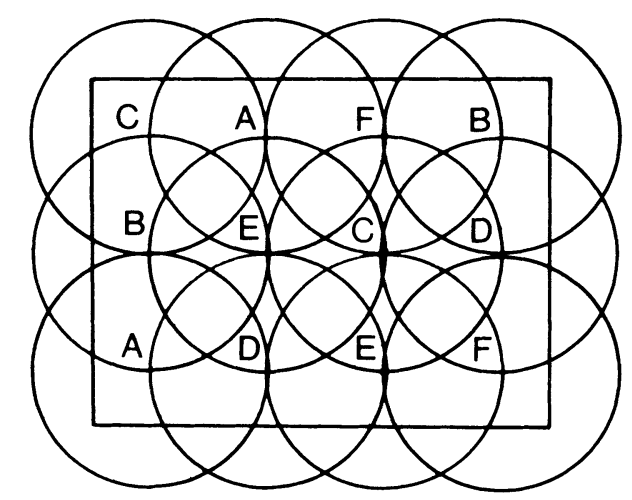

$$
\begin{array}{ll}
w_{1}=4-i & A, B, E, F: \text { Reflection in a vertical plane } \\
w_{2}=3 i & C, D: \text { Reflection in a horizontal plane }
\end{array}
$$

FIGURE 18

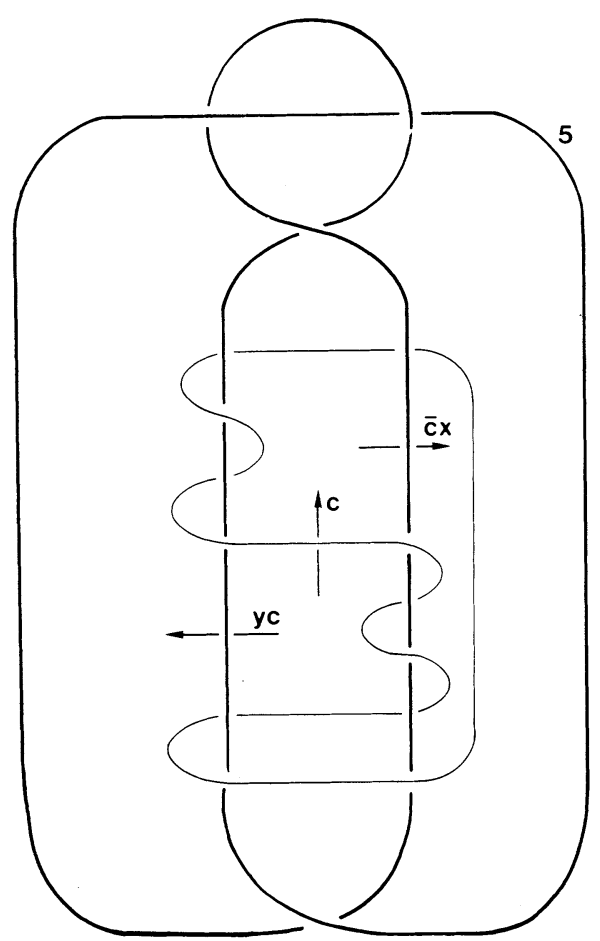

FIGURE 19 
G. A link in a mapping torus of a homeomorphism $h$ of a torus where $h$ is given by $\left(\begin{array}{cc}0 & 1 \\ -1 & -1\end{array}\right)$.

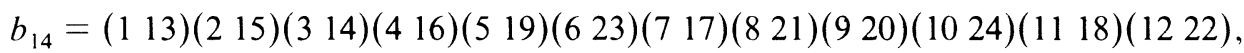

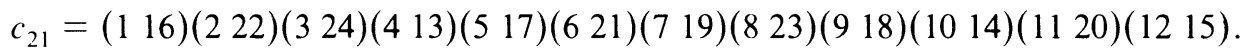

A presentation is $\left\langle u, v, w ; u w v^{2} \bar{u} v w^{2}, w \bar{u} \bar{w}^{2} \bar{v}^{2} w \bar{u} \bar{w}^{2} \bar{u} v w^{2} v\right\rangle$ where

$$
u=\left(\begin{array}{cc}
1 & 2-2 i \\
0 & 1
\end{array}\right), \quad v=\left(\begin{array}{cc}
1 & 1+2 i \\
i & i-1
\end{array}\right), \quad w=\left(\begin{array}{cc}
0 & i \\
i & -i
\end{array}\right) .
$$

The peripheral subgroup is $\left\{u, \bar{w}^{2} \bar{v}^{2} w u v^{2}\right\}$, with meridian $u$ and $\tau$-invariant $\frac{3}{2} i$. The Ford domain is given in Figure 20 and the link in Figure 21. The fundamental group for the mapping torus has a presentation $\left\langle v, w ;\left(v w^{2}\right)^{3}=w^{3}=\bar{v}^{3}\right\rangle$; we have shown the manifold in the form obtained by three -3 surgeries and one -1 surgery on a link of four components. The generators for the fundamental group are shown in the figure.

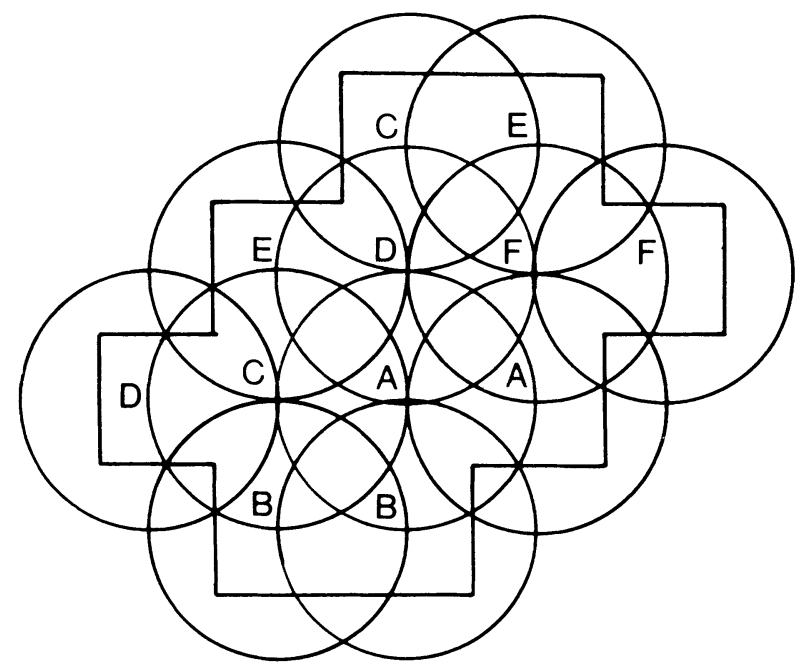

$$
\begin{array}{ll}
w_{1}=2-2 i & A, B, F: \text { Reflection in a horizontal plane } \\
w_{2}=3+3 i & C, D, E: \text { Reflection in a vertical plane }
\end{array}
$$

FIGURE 20

H.1. A link in the lens space $L(3,1)=$ Double cover of $S^{3}-W g$ branched along the trefoil.

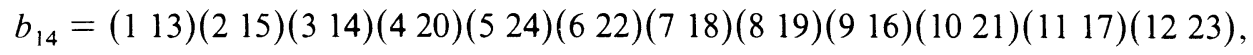

$$
\begin{aligned}
& c_{21}=\left(\begin{array}{ll}
1 & 14
\end{array}\right)(221)(313)(419)(522)(624)(723)(820)(917)(1015)(1116)(1218) \text {. }
\end{aligned}
$$

A presentation is $\left\langle u, v, w ; w u w^{2} v^{2} u v u=1, v u v^{2} w^{2} u w u=1\right\rangle$ where

$$
u=\left(\begin{array}{cc}
-i & 2 i \\
-1+i & 2-i
\end{array}\right), \quad v=\left(\begin{array}{cc}
-i & -1 \\
1 & 0
\end{array}\right), \quad w=\left(\begin{array}{cc}
-1 & -1+2 i \\
i & 1+i
\end{array}\right)
$$




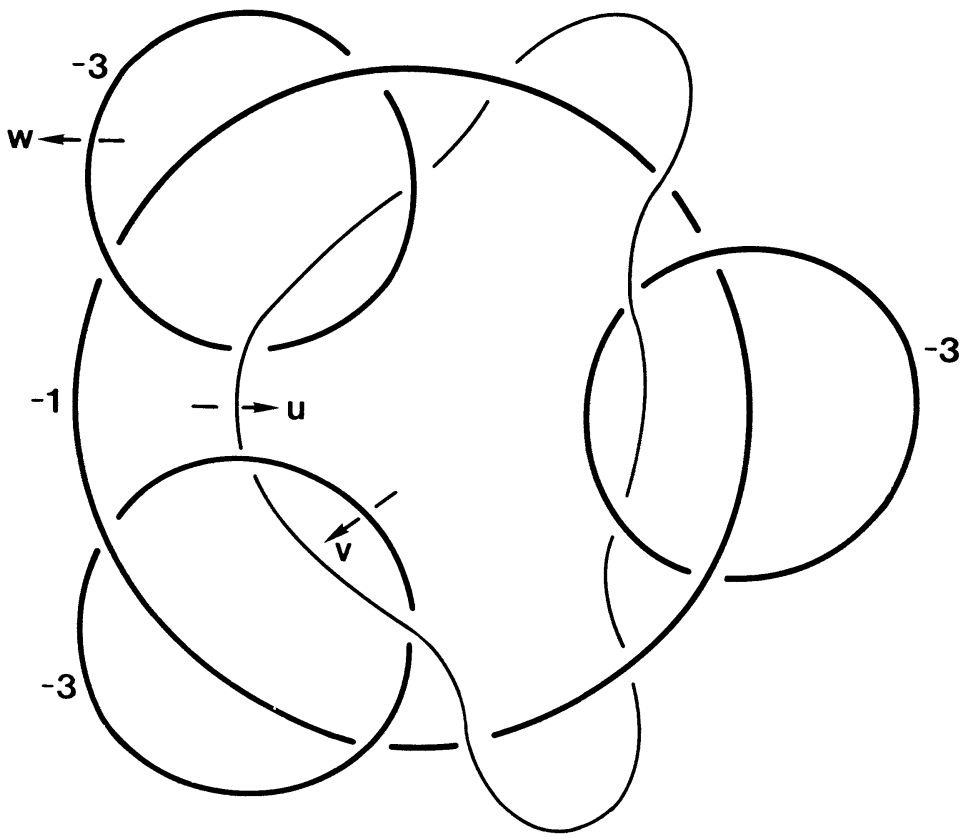

FIGURE 21

The peripheral subgroups are $\left\{v^{2} w, v^{2} u v u\right\}$ and $\left\{w^{2} v, w^{2} u w u\right\}$ both with $\tau$-invariant $i$. The Ford domain is given in Figure 22 and the link in Figure 23. The manifold was obtained from the 2-fold double cover of $S^{3}-W g$ branched along the trefoil, by deleting the preimage of the link under the covering projection.

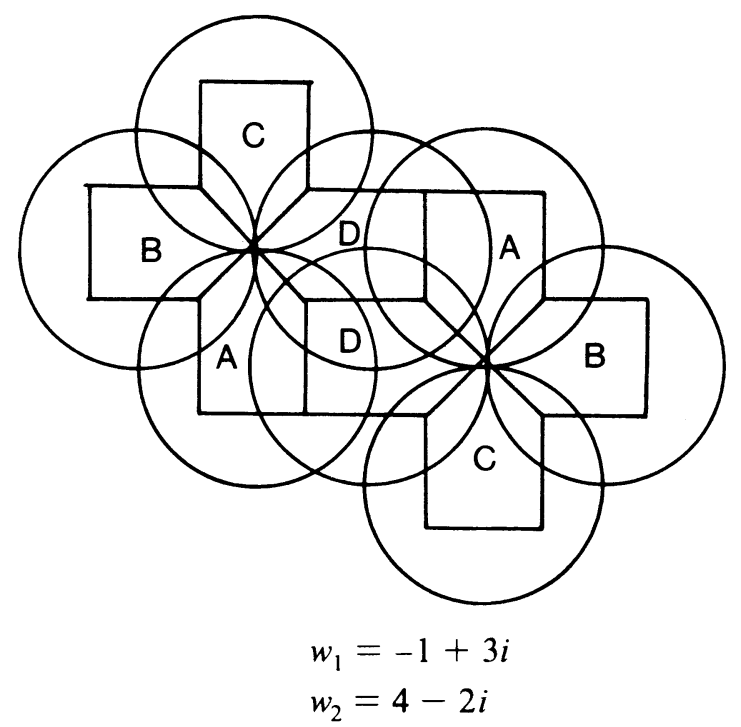

Figure 22 


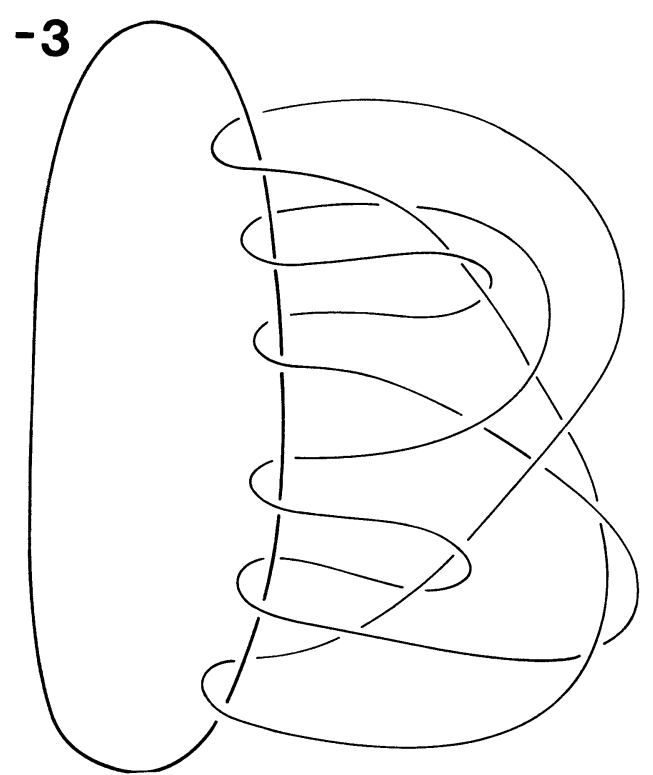

FIgURE 23

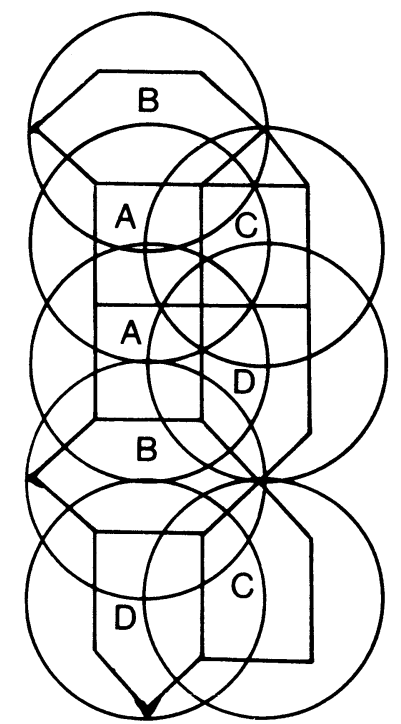

$$
\begin{array}{ll}
w_{1}=2 & A: \text { Reflection in a vertical plane } \\
w_{2}=1-5 i & B: \text { Reflection in a horizontal plane }
\end{array}
$$

Figure 24

H.2. A link in $S^{1} \times S^{2}$.

$$
\begin{aligned}
& b_{14}=\left(\begin{array}{ll}
1 & 13
\end{array}\right)(215)(314)(417)(516)(620)(721)(818)(919)(1023)(1122)(1224), \\
& c_{21}=\left(\begin{array}{ll}
1 & 14
\end{array}\right)(2 \text { 20)(3 13)(4 19)(5 22)(6 15)(7 18)(8 21)(9 17)(10 24)(11 16)(12 23). }
\end{aligned}
$$

A presentation is $\left\langle u, v, w ; \bar{w} \bar{v}^{2} u v^{2} w=\bar{v} \bar{u} v \bar{u}, \bar{w} u w=\bar{v} \bar{u} v \bar{v}^{2} \bar{u} v^{2}\right\rangle$ where

$$
u=\left(\begin{array}{cc}
1 & -2 \\
0 & 1
\end{array}\right), \quad v=\left(\begin{array}{cc}
-i & -1 \\
1 & 0
\end{array}\right), \quad w=\left(\begin{array}{cc}
-1+2 i & 2 i \\
1+i & 1
\end{array}\right)
$$


One peripheral subgroup is $\left\{w u, \bar{u} \bar{v}^{2} u v^{2}\right\}$ with $\tau$-invariant $i$, and the other is $\left\{u, v^{2} w u v \bar{w}\right\}$ with $\tau$-invariant $\frac{1}{2}+\frac{5}{2} i$. The Ford domain is given in Figure 24 and the link in Figure 25.

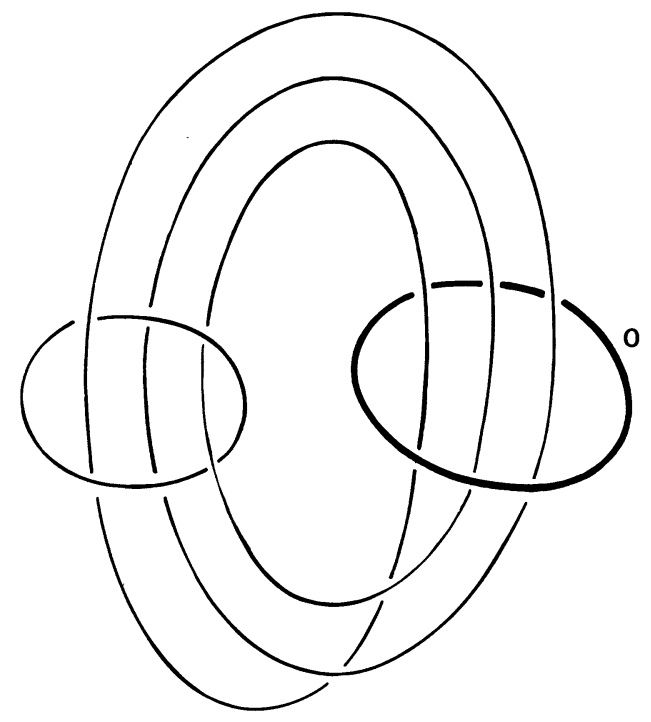

Figure 25

I.1. A link in $S^{1} \times S^{2}$.

$b_{14}=\left(\begin{array}{ll}1 & 13\end{array}\right)(215)(314)(412)(519)(69)(711)(821)(1024)(1620)(1723)(1822)$,

$c_{21}=\left(\begin{array}{ll}1 & 7\end{array}\right)(25)(317)(410)(620)(818)(916)\left(\begin{array}{lll}11 & 13\end{array}\right)(1224)(1423)(1519)(2122)$.

A presentation is $\left\langle u, v, w ; w \sim u^{2} \bar{v}^{2}, \bar{w} \bar{u} v w=u^{3} \bar{v}^{3}\right\rangle$ where

$$
u=\left(\begin{array}{cc}
i & 1 \\
-1+i & 1
\end{array}\right), \quad v=\left(\begin{array}{cc}
-i & -1 \\
1+i & 1
\end{array}\right), \quad w=\left(\begin{array}{cc}
1 & 2 i \\
0 & 1
\end{array}\right), \quad u^{2} \bar{v}^{2}=\left(\begin{array}{ll}
1 & 2 \\
0 & 1
\end{array}\right) .
$$

One peripheral subgroup is $\left\{w, u^{2} \bar{v}^{2}\right\}$ with $\tau$-invariant $i$, and the other is $\left\{\bar{u} v, w u w u^{3}\right\}$ with $\tau$-invariant $2 i$. The Ford domain is given in Figure 26 and the link in Figure 27.

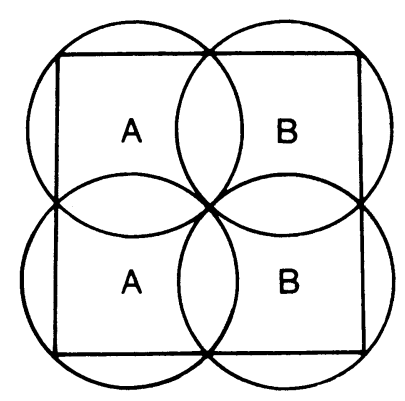

$A:+\pi / 2$ rotation follows reflection in a horizontal plane

$B$ : $-\pi / 2$ rotation follows reflection in a horizontal plane

FigURE 26 


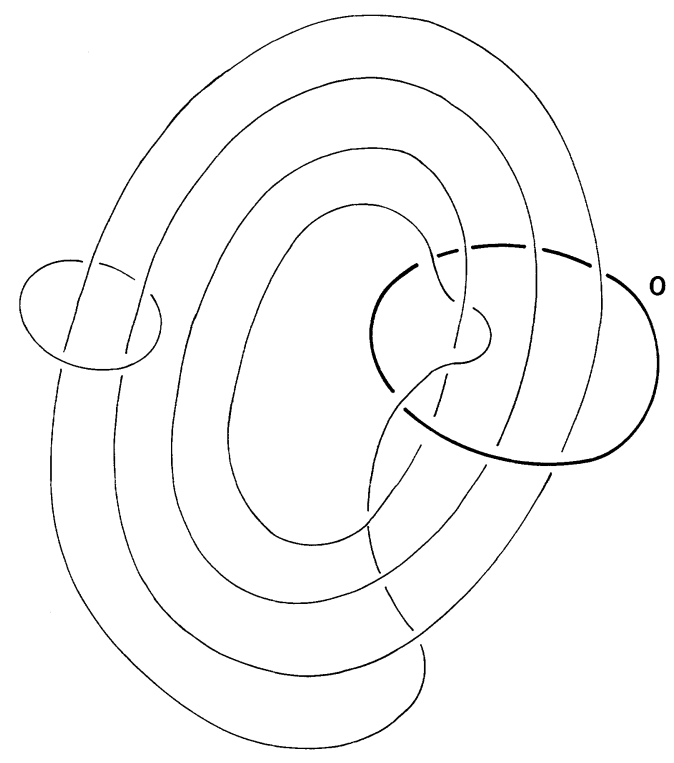

Figure 27

I.2. A link in the lens space $L(8,3)=$ double cover of $S^{3}-W d$ branched along both components.

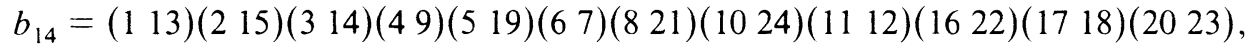

$$
\begin{aligned}
& c_{21}=\left(\begin{array}{ll}
1 & 7
\end{array}\right)(25)(320)(4 \text { 10)(6 13)(8 16)(9 24)(11 18)(12 17)(14 23)(15 19)(21 22). }
\end{aligned}
$$

A presentation is $\left\langle u, v, w ; w \sim u^{2} \bar{v} \bar{w} v, \bar{w} \bar{u} v^{3} w=u^{3} \bar{v}\right\rangle$ where

$$
u=\left(\begin{array}{cc}
i & 1 \\
-1+i & 1
\end{array}\right), \quad v=\left(\begin{array}{cc}
1 & 1 \\
-1+i & i
\end{array}\right), \quad w=\left(\begin{array}{cc}
1 & 2 i \\
0 & 1
\end{array}\right), \quad u^{2} \bar{v} \bar{w} \bar{v}=\left(\begin{array}{ll}
1 & 2 \\
0 & 1
\end{array}\right) .
$$

One peripheral subgroup is $\left\{w, u^{2} \bar{v} \bar{w} \bar{v}\right\}$ with $\tau$-invariant $i$ and the other is $\{v w v \bar{u} v$, $\left.\bar{v} u w v^{2}\right\}$ with $\tau$-invariant $i$. The Ford domain is given in Figure 28 and the link in Figure 29.

An $\frac{8}{3}$ Dehn surgery is done along the trivial knot. The manifold is obtained as the double cover of $S^{3}-W d$ branched along both components with the branching set deleted.

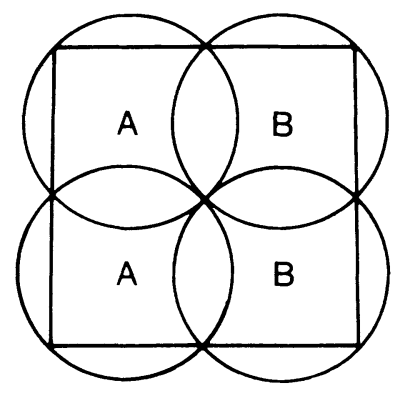

$A, B:-\pi / 2$ rotation follows reflection in a horizontal plane

FIGURE 28 


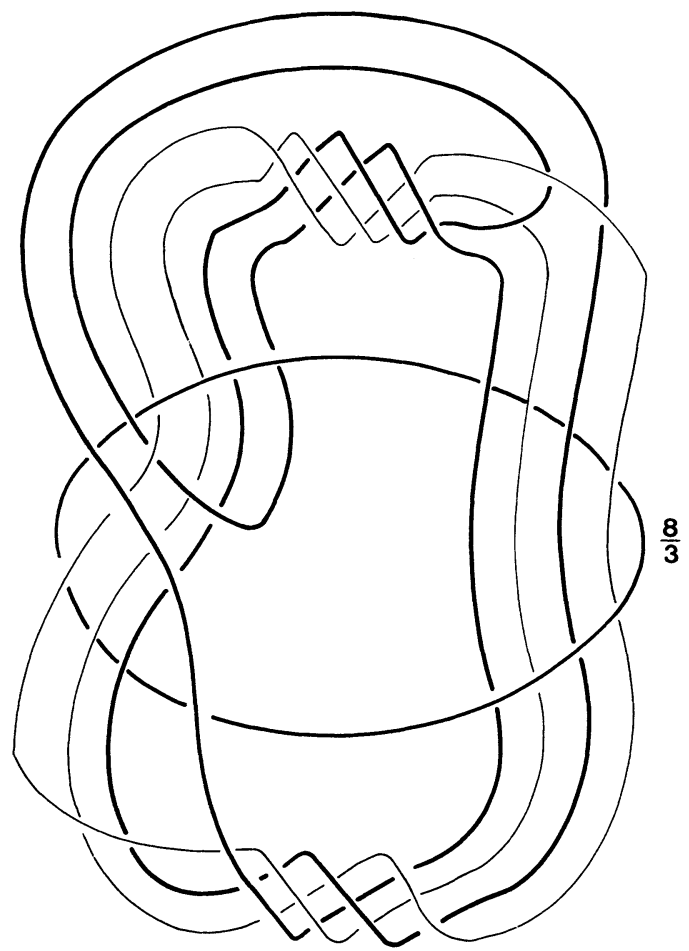

Figure 29

J. A link in $S^{1} \times S^{2}$.

$b_{14}=(19)(212)(37)(410)(56)(811)(1318)(1423)(1516)(1719)(2021)(2224)$,

$c_{21}=\left(\begin{array}{ll}1 & 18\end{array}\right)(223)(316)(420)(524)(622)(7$ 15)(8 19)(9 13)(10 21)(11 17)(12 14).

A presentation is $\left\langle u, v, w ; w u^{2} v^{2} \bar{w}=\bar{v}^{3} \bar{u}^{3}, u v \sim v^{2} w v\right\rangle$ where

$$
u=\left(\begin{array}{cc}
-1-2 i & i \\
-1-i & i
\end{array}\right), \quad v=\left(\begin{array}{cc}
-3 i & 3 i-2 \\
-1-3 i & 4 i-1
\end{array}\right), \quad w=\left(\begin{array}{cc}
-2 & 1+2 i \\
2 i-3 & 3+2 i
\end{array}\right) .
$$

The two peripheral subgroups are $\left\{u v, v^{2} w v\right\}$ and $\left\{u^{2} v^{2}, w v w\right\}$ both with $\tau$-invariant $\frac{3}{2} i$. The Ford domain is given in Figure 30 and the link in Figure 31 .

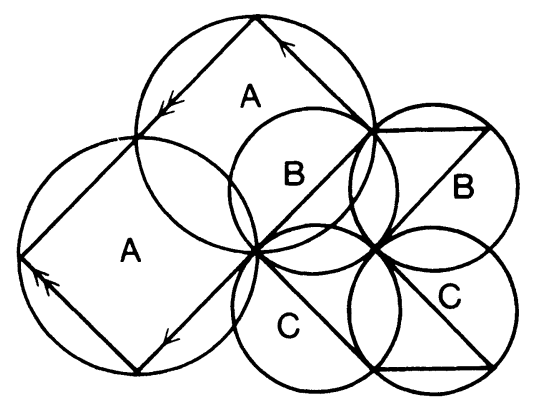

$w_{1}=2 i \quad A:$ Reflection in horizontal plane

$w_{2}=3 \quad B, C$ : Take triangular face to triangular face

FIGURE 30 


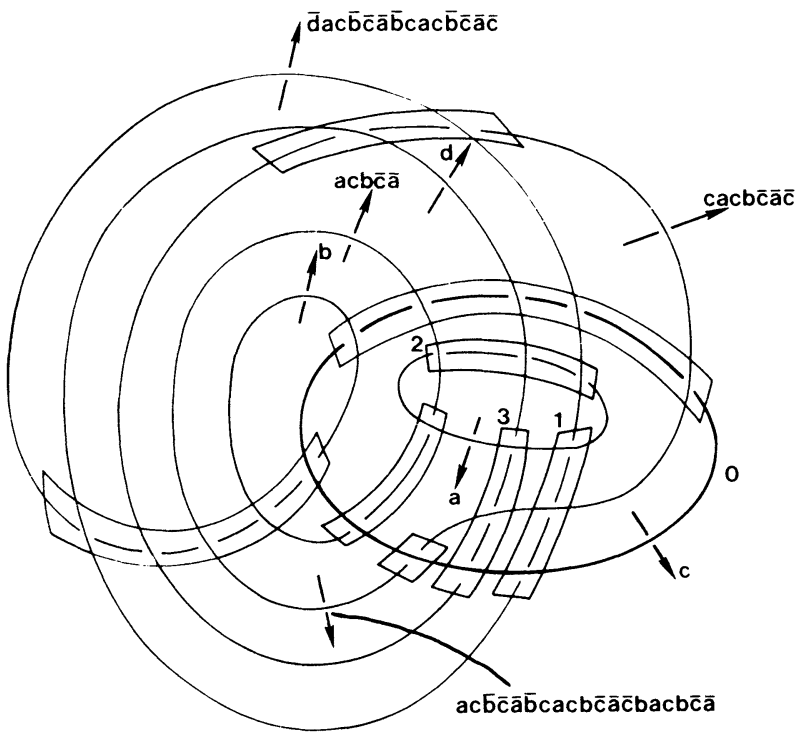

Figure 31

We verify that the manifold above has the correct fundamental group. The fundamental group is generated by $a, b, c$ and $d$, as given in the figure, together with the following relations:

1. $a c a c b \bar{c} \bar{a}$ dac $\bar{b} \bar{c} \bar{a} \bar{c} \bar{a} a c b \bar{c} \bar{a} \bar{c} b a c b \bar{c} \bar{a} d=1$

2. $a \sim c a c b \bar{c} \bar{a} \bar{c} b$

3. $d=a c \bar{b} c a c b \bar{c} \bar{a} \bar{c} b \bar{c} \bar{a}$.

The relations arising from other rectangles and the frame 0 surgery were already used when eliminating a number of generators.

Eliminating $d$, we have

1. $a c a c^{2} a c b \bar{c} \bar{a} \bar{c}^{2} \bar{a} \bar{c} \bar{a} c a c b \bar{c} \bar{a} \bar{c} b a c^{2} a c b \bar{c} \bar{a} \bar{c} b \bar{c} \bar{a}=1$

2. $a \sim c a c b \bar{c} \bar{a} \bar{c} b$.

Reducing the first relation using the second gives

1. $a c^{2} a c b \bar{c} \bar{a} \bar{c} b \bar{c} \bar{a} \bar{c} b c^{2} a c b \bar{c} \bar{a} \bar{c} b=1$

2. $a \sim c a c b \bar{c} \bar{a} \bar{c} b$.

Now, introducing new generators, $a=\bar{w} \bar{v} \bar{w}, b=\bar{v} \bar{u}, c=w$ we obtain

1. $w u^{2} v^{2} \bar{w}=\bar{v}^{3} \bar{u}^{3}$

2. $w v w \sim u^{2} v^{2}$.

It is enough, then, to show that $u v \sim v^{2} w v$ is a consequence. But,

$$
\begin{aligned}
\bar{v} \bar{w} \bar{v}^{2} u v^{3} w v & =\bar{v} \bar{w} \bar{v}^{2} \bar{u}^{2} w \bar{v}^{2} \bar{u}^{2} v, & \text { using } 1 \\
& =w \bar{v}^{2} \bar{u}^{2} \bar{w} \bar{v}^{3} \bar{u}^{2} v, & \text { using } 2 \\
& =u v, \quad \text { using } 1 . &
\end{aligned}
$$

11. Appendix. In this section, we verify that the manifolds given by surgery instructions in $\$ 10$ are homeomorphic to the hyperbolic 3-manifolds corresponding to our subgroups. 
1. It is well known that the Borromean rings correspond to the subgroups in B.2. The manifolds corresponding to A.1, B.1, H.1, and I.2 are obtained as double covers of the two hyperbolic 3-manifolds, $S^{3}-W g$ and $S^{3}-W d$ studied in $\S \S 6$ and 7; the surgery instructions can easily be obtained using the standard techniques for studying double covers. The trick is to unknot the branching sets by introducing framed surgeries.

2. Let $N$ be one of the manifolds not considered in 1 above, with $H$ the corresponding subgroup, and let $M$ be the closure of the hyperbolic 3-manifold corresponding to $H$. It can be verified algebraically, with some effort, that there is an isomorphism $\psi$ from $\pi_{1}(N)$ to $\pi_{1}(M)=H$. According to Waldhausen (Corollary 6.5 of [9]), $\psi$ can be realized by a homeomorphism provided that $\psi$ respects the peripheral structures, $N$ is irreducible, and also boundary irreducible. Any isomorphism from $\pi_{1}(N)$ to $\pi_{1}(M)$ has to respect the peripheral structure because $M$ is a hyperbolic manifold whose boundary consists of tori. Also, the existence of $\psi$ implies that $N$ is boundary irreducible, because $M$ is. Therefore, it remains to show that $N$ is irreducible.

Lemma. Suppose that $N$ is the closure of a link complement in a closed 3-manifold $W$ and $\pi_{1}(N)$ is isomorphic to one of our subgroups. Suppose also that $W$ does not contain a fake 3-ball. If $S^{2} \subset N$ is an embedded 2-sphere, and $\left[S^{2}\right]=0$ in $\pi_{2}(W)$ or $\mathrm{H}_{2}(\mathrm{~W} ; \mathbf{Z})$, then $\mathrm{S}^{2}$ bounds a 3-ball in $N$.

Proof. Since [ $\left.S^{2}\right]=0$ in $H_{2}(W)$, it follows that $S^{2}$ splits $W$ into two components. Thus, $S^{2}$ also splits $N$ into two components; say $N=N_{1} \cup N_{2}$, where $N_{1}$ and $N_{2}$ are identified along $S^{2}$. Since none of our subgroups can be a nontrivial free product, we may assume that $\pi_{1}\left(N_{1}\right)=\{1\}$. Furthermore, the boundary of $N$ is contained in $N_{2}$ since the inclusion homomorphism of $\pi_{1}(\partial N)$ into $\pi_{1}(N)$ is injective. Therefore, $\partial N_{1}=S^{2}$ and $N_{1}$ (which is contained in $W$ ) is a contractible 3-manifold. It follows that $N_{1}$ is homeomorphic to the 3-ball since $W$ does not contain a fake 3-ball. This completes the proof of the lemma.

We now divide the argument into cases.

(a) (Manifolds A.2, A.3, B.2, B.3, C). The lemma applies, since $H_{2}\left(S^{3}\right)=0$ and $S^{3}$ does not contain a fake 3-ball.

(b) (Manifold D). The Poincare homology 3-sphere $W$ is covered by $S^{3}$; hence $W$ cannot contain a fake 3-ball, and $H_{2}(W)=H_{1}(W)=0$.

(c) (Manifolds E.1, E.2, G). It can be shown that the closed manifold $W$ in E.2 can be doubly covered by a 0 -frame surgery on the connected sum of two figure 8 knots. A connected sum of two figure 8 knots is fibred, and, in general, 0 -surgery on a fibred knot produces a surface bundle over $S^{1}$. Therefore, the universal cover of $W$ is $R^{3}$, and so the lemma applies. As the closed manifold in E. 1 is obtained by 0 -surgery on the fibred knot $10_{153}$, the above argument works in this case also.

The same considerations also apply to $G$, where the closed manifold can be seen to be homeomorphic to the mapping torus corresponding to the homeomorphism of order 3 of a torus given by $\left(\begin{array}{cc}0 & 1 \\ -1 & -1\end{array}\right)$. 
(d) (Manifold F) Here the closed manifold is 5-surgery on the figure 8 knot. It is shown in Thurston [8] that this manifold is hyperbolic, and thus it is irreducible. Hence the lemma applies.

(e) (Manifolds H.2, I.1, J). Let $N$ be one of these manifolds. Then $N=$ closure of $(W-L)$ where $W=S^{1} \times S^{2}$. In each of the cases a component of the link $L$ represents a nontrivial element in $H_{1}(W)$. Thus, if $S^{2} \subset N$, then since $S^{2}$ and $L$ do not intersect, duality implies that $\left[S^{2}\right]=0$ in $H_{2}(W)$. The lemma now applies since $S^{1} \times S^{2}$ does not contain a fake 3-ball.

Note added in proof. An alternative method for finding presentations of subgroups and their (common) fundamental domains can be found in $A$ geometric method for presenting subgroups of discrete groups, by Y. W. Lee, which is to appear in Topology and its Applications.

\section{BIBLIOGRAPHY}

1. A. Karrass and D. Solitar, The subgroups of a free product of two groups with an amalgamated subgroup, Trans. Amer. Math. Soc. 150 (1970), 227-255.

2. A. Karrass, A. Pietrowski and D. Solitar, The subgroups of a polygonal product of groups (to appear).

3. W. Magnus, A. Karrass and D. Solitar, Combinatorial group theory, Pure and Appl. Math., vol. 13, Interscience, New York, 1966.

4. R. Riley, Discrete parabolic representations of link groups, Mathematika 22 (1975), 141-150.

5. S__ Seven excellent knots, Low-Dimensional Topology, (Proc. Conf. Topology in Low Dimensions, 1979), Cambridge Univ. Press, Cambridge, 1982.

6. D. Rolfsen, Knots and links, Publish or Perish, Boston, Mass. 1976.

7. R. G. Swan, Generators and relations for certain special linear groups, Adv. in Math. 6 (1970), 1-77.

8. W. Thurston, The geometry and topology of 3-manifolds, Princeton Univ. Press, Princeton, N.J., 1982.

9. F. Waldhausen, On irreducible 3-manifolds which are sufficiently large, Ann. of Math. (2) 87 (1968), $56-88$.

10. N. Wielenberg, Hyperbolic 3-manifolds which share a fundamental polyhedron, Riemann Surfaces and Related Topics, (Proc. the 1978 Stony Brook Conference), Princeton Univ. Press, Princeton, N.J., 1980.

Science Division, The University of Wisconsin-Parkside, Kenosha, Wisconsin 53141

Department of Mathematics, New College of the University of South Florida, Sarasota, FLORIDA 33576 